To be published in The Astrophysical Journal

\title{
A Fresh Catch of Massive Binaries in the Cygnus OB2 Association
}

\author{
Henry A. Kobulnicky ${ }^{1}$, Rachel A. Smullen ${ }^{1}$, Daniel C. Kiminki ${ }^{2}$, Jessie C. Runnoe ${ }^{1}$, Earl S. \\ Wood $^{1}$, Garrett Long ${ }^{1}$, Michael J. Alexander ${ }^{1}$, Michael J. Lundquist ${ }^{1}$, Carlos \\ Vargas-Alvarez ${ }^{1}$
}

\begin{abstract}
Massive binary stars may constitute a substantial fraction of progenitors to supernovae and $\gamma$-ray bursts, and the distribution of their orbital characteristics holds clues to the formation process of massive stars. As a contribution to securing statistics on OB-type binaries, we report the discovery and orbital parameters for five new systems as part of the Cygnus OB2 Radial Velocity Survey. Four of the new systems (MT070, MT174, MT267, and MT734三VI Cygni \#11) are single-lined spectroscopic binaries while one (MT103) is a double-lined system $(\mathrm{B} 1 \mathrm{~V}+\mathrm{B} 2 \mathrm{~V})$. MT070 is noteworthy as the longest period system yet measured in Cyg OB2, with $P=6.2$ yr. The other four systems have periods ranging between 4 and 73 days. MT174 is noteworthy for having a probable mass ratio $q<0.1$, making it a candidate progenitor to a low-mass X-ray binary. These measurements bring the total number of massive binaries in Cyg OB2 to 25, the most currently known in any single cluster or association.
\end{abstract}

Subject headings: Techniques: radial velocities — (Stars:) binaries: general — (Stars:) binaries: spectroscopic — (Stars:) binaries: (including multiple) close — Stars: early-type — Stars: kinematics and dynamics

\section{Introduction}

Massive stars produce some of nature's most energetic phenomena as they end their lives in spectacular supernova or $\gamma$-ray burst explosions (Burrows et al. 1995; Woosley et al.

\footnotetext{
${ }^{1}$ Dept. of Physics \& Astronomy, University of Wyoming, Laramie, WY 82070 chipk@uwyo.edu

${ }^{2}$ Dept. of Astronomy, University of Arizona, Tucson, AZ 85721
} 
1993; Woosley \& Bloom 2006). While the post-main-sequence evolutionary path of a single massive star is still not settled (e.g., the sequence through red/blue supergiant, Wolf-Rayet, Luminous Blue Variable phases for stars of various masses; Maeder \& Meynet 2000), the possible evolutionary paths of close massive binary systems are even less certain. Nevertheless, close massive binaries appear common (Garmany et al. 1980; Sana \& Evans 2011; Kiminki et al. 2012), and secondary stars in such systems have been implicated in removing hydrogen envelopes from massive primaries prior to core collapse, resulting in a substantial fraction (30-40\%) of Type Ib/c supernovae and $\gamma$-ray bursts (Izzard et al. 2004; Kobulnicky \& Fryer 2007; Eldridge et al. 2008). Close massive binaries may also produce the observed population of high-velocity "runaway" stars either through ejection of one component after the supernova explosion or during multi-body gravitational interactions (Gies \& Bolton 1986; Blaauw 1961).

Papers I-IV in this series (Kiminki et al. 2007, Kiminki et al. 2008, Kiminki et al. 2009, Kiminki et al. 2012) describe prior results of the Cygnus OB2 Radial Velocity Survey intended to measure the massive binary characteristics (i.e., binary fraction, distribution of periods, mass ratios, eccentricities) for a large number (114) massive stars in a single cluster/association having a common formation environment. In particular, Paper IV (Table 6) summarizes orbital elements for the 20 massive binaries with measured parameters. Paper V (Kiminki \& Kobulnicky 2012) uses these previously published data and the new data presented herein (a total of 25 measured systems) to infer the intrinsic distributions of massive binaries, concluding that the fraction of massive stars (defined as B3 and earlier; i.e., supernova progenitors 1 ) having companions may be as high as $90 \%$, and that $45 \%$ of these are likely to interact at some point. Paper $\mathrm{V}$ reports an excess of short-period 4-7 day systems relative to $7-14$ day systems and finds that the distribution of mass ratios is approximately flat over the range $0.1<q<1.0$. Besides providing the fundamental data for modeling the frequencies of energetic phenomena, these types of statistics help place constraints on theoretical frameworks for the formation of massive stars which remain under debate (Krumholz et al. 2010; Smith et al. 2009).

In this sixth paper of the series we present orbital solutions for five additional massive spectroscopic binaries in Cyg OB2 - one double-lined (SB2) and four single-lined (SB1) systems - using spectroscopic data obtained primarily during 2010-2011, but utilizing some data as early as 1999. Using the nomenclature of Massey \& Thompson (1991), these systems are MT103 (SB2), MT070, MT174, MT267, and MT734 (SB1s). Figure 1 displays a three-color representation of the Cyg OB2 vicinity, where blue/green/red depict the Palo-

\footnotetext{
${ }^{1}$ For a typical initial mass function (Salpeter 1955), B stars outnumber O stars 3:1 as supernova progenitors.
} 
mar Sky Survey R, the Spitzer $4.5 \mu \mathrm{m}$, and the Spitzer $8.0 \mu \mathrm{m}$ bands, respectively. White labels denote previously known binary systems, while magenta labels highlight the newly discovered systems reported herein. Numeration follows the system of Massey \& Thompson (1991), with "S" additionally indicating the numeration of Schulte (1958) and "A" or "B" for

that of Comerón et al. (2002). Figure 1 shows the complex nature of this region, including ionized gas (diffuse blue emission tracing $\mathrm{H} \alpha$ within the POSS R band), photo-dissociation regions (PDR) at the edges of molecular clouds (diffuse red and green tracing broad emission features arising from excited polycyclic aromatic hydrocarbons, PAHs), and stars (blue and green point sources).

After first reviewing the Survey strategy and data, we present the new radial velocity data and describe the orbital characteristics of these $\mathrm{O}$ and early $\mathrm{B}$ systems. We conclude by summarizing the parameters of the 25 currently known massive binaries in CygOB2. All reported velocities are in the heliocentric frame unless explicitly indicated otherwise.

\section{Spectroscopic Observations, Reductions, and Radial Velocity Measurement}

The Cygnus OB2 Radial Velocity Survey is an ongoing optical spectroscopic survey of 120 massive primary stars (earlier than B3) drawn from the Massey \& Thompson (1991) photometric study of Cyg OB2. Papers I-IV provide details regarding the variety of observatories, dates of observation, and data analysis methods employed dating back to the start of the survey in 1999. Observing cadences vary from nightly to as few as several observations per year for the survey overall. Here, we repeat essential observational details and report on new observations covering the 2010 and 2011 observing seasons at the Wyoming Infrared Observatory (WIRO) $2.3 \mathrm{~m}$ telescope. Table 1 provides a log of observation dates and derived radial velocities for the SB2 MT103 and Table 2 lists observing dates and velocities for the four SB1 systems.

In 2011, the data were obtained using the WIRO Longslit spectrograph with an e2V $2048^{2}$ ccd as the detector. An $18001 \mathrm{~mm}^{-1}$ grating in first order yielded a spectral resolution of $1.5 \AA$ near $5800 \AA$ with a $2^{\prime \prime} \times 100^{\prime \prime}$ slit. The spectral coverage was 5250-6750 . Exposure times ranged from $1200 \mathrm{~s}$ to $3600 \mathrm{~s}$ in multiples of $600 \mathrm{~s}$ depending on target brightness, current seeing (1.2-3 arcseconds FWHM) and cloud conditions. Reductions followed standard longslit techniques, including flat fielding from dome quartz lamp exposures. Copper-argon arc lamp exposures were taken after each star exposure to wavelength calibrate the spectra to an rms of $0.03 \AA$ (1.5 $\mathrm{km} \mathrm{s}^{-1}$ at $\left.5800 \AA\right)$. Multiple exposures were combined yielding final $\mathrm{S} / \mathrm{N}$ ratios typically in excess of 100:1 near $5800 \AA$. Final spectra were Doppler corrected to the heliocentric frame. Each spectrum was then shifted by a small additional amount in 
velocity so that the Na I D $\lambda \lambda 5890,5996$ lines were registered with the mean Na I line wavelength across the ensemble of observations. This zero-point correction to each observation is needed to account for effects of image wander in the dispersion direction when the stellar FWHM of the point spread function was appreciably less than the slit width. Because of these inevitable slit-placement effects on the resulting wavelength solutions at the level of $\lesssim 10 \mathrm{~km} \mathrm{~s}^{-1}$, radial velocity standards were not routinely taken. Relative velocity shifts were generally less than $7 \mathrm{~km} \mathrm{~s}^{-1}$.

In 2010 the WIRO-Longslit data were obtained with the $600 \mathrm{l} \mathrm{mm}^{-1}$ grating in second order covering 4000-5900 $\AA$ at $2.8 \AA$ FWHM resolution near $5800 \AA$. Reductions followed the same procedures previously described. The lower spectral resolution of these data limited their utility and are seldom used, but we note them here for completeness.

We measured the radial velocity for each spectrum obtained at WIRO using Gaussian fits to the He I $\lambda 5876$ line. Systemic velocities are, therefore, based solely on this line, by adopting a rest wavlength of $5875.69 \AA$ measured in model stellar spectra with static atmospheres (TLUSTY; Lanz \& Hubeny 2003; Hubeny \& Lanz 1995). Stars with strong winds, including very early O stars (not present in this particular subsample) and evolved stars (MT267, MT734) may exhibit He line centers that are blue shifted with respect to this assumed rest wavelength. Our fitting code 2 fixes the Gaussian width and depth to be the mean determined from all the spectra, after rejecting outliers, and it solves for the best fitting line center. In the case of an SB2 like MT103, the code fits a double Gaussian where the widths and depths have been fixed independently using observations obtained near quadrature. In the case of the long-period system MT070 (6.2 years) we included data from the WIYN telescope \& Hydra fiber spectrograph covering the range 3800-4500 $\AA$. Therefore, we measured radial velocities by fits to the He I $\lambda 4471$ line instead of $\lambda 5876$. Our analysis of Keck spectra of stars having minimal radial velocity variations suggest no systematic differences between these two lines. However, the velocities from the WIYN/Hydra appear systematically $5-15 \mathrm{~km} \mathrm{~s}^{-1}$ more negative than velocities from WIRO for constant-velocity systems having sufficient data from both observatories to make this comparison. Observations of radial velocity standard stars using WIYN/Hydra showed excellent agreement with published velocities (Paper I). Given the spectral stability of the fiber-fed Hydra bench-mounted spectrograph, we consider velocities from this instrument to be more reliable. Accordingly, when WIRO and WIYN spectra are combined to compute solutions for the same star (MT070), we shift the WIRO velocities by $-10 \mathrm{~km} \mathrm{~s}^{-1}$. We caution that this may introduce systematic shifts in the systemic velocity reported. However, the primary goal of the Cygnus OB2 radial velocity

\footnotetext{
${ }^{2}$ We use the robust curve fitting algorithm MPFIT as implemented in IDL (Markwardt 2009).
} 
survey is to obtain orbital parameters for massive binaries, a goal that requires good relative radial velocities. Absolute space velocities from which systemic velocities may be inferred are of lesser importance. Tables 1 and 2 report the adopted radial velocities and uncertainties for each star, along with the orbital phase, $\phi$, from the best-fitting solution, and the observed minus computed $(\mathrm{O}-\mathrm{C})$ residuals. The final column specifies the observatory used to obtain the data.

\section{Orbital Solutions}

We analyzed the CLEANed (Roberts et al. 1987) power spectrum for each object to select likely periods and then examined the folded velocity curve for periods corresponding to the strongest peaks in the power spectrum. In most cases the strongest peaks yielded clean, convincing phased velocity curves. Secondary peaks and possible aliases could be eliminated by visual inspection owing to the much larger dispersion in the data at any given phase. We used the binary orbital solution package "binary" by D. Gudehus 3 with these initial period estimates and the radial velocity data to solve for the full suite of orbital parameters and associated uncertainties. Table 3 compiles these best-fitting parameters and uncertainties for each object. Listed within the table are the period in days $(P)$, eccentricity of the orbit $(e)$, longitude of periastron in degrees $(\omega)$, systemic radial velocity $(\gamma)$, epoch of periastron $\left(T_{0}\right)$, primary and secondary (for MT103) projected semi-amplitudes $\left(K_{1} \&\right.$ $K_{2}$ ), spectral classifications from this survey (S.C.1 \& S.C.2), estimates of the mass ratio $(q)$, inclination $(i)$, semi-major axis $(a)$, and reduced chi squared values of the best fitting solution. The ensuing subsections elaborate on the detailed orbital solutions for the five massive systems.

\subsection{MT070}

MT070 has $V=12.99$ with a systemic velocity of $-6.4 \mathrm{~km} / \mathrm{s}$, as reported by Kiminki et al. (2007). Its $B-V$ color of 2.10 mag makes it among the reddest early-type stars in Cyg OB2. Figure 1 show that MT070 lies along the eastern edge of the Association (upper right) in an apparently unremarkable region mostly devoid of $\mathrm{H} \alpha$ and $\mathrm{PAH}$ emission. However, there are filaments of $8 \mu \mathrm{m} \& 24 \mu \mathrm{m}$ emission and ${ }^{12} \mathrm{CO}$ (C. Brunt, private communication) at Cyg OB2 radial velocities cutting across this region, suggesting the likelihood of elevated dust extinction that could explain the redder color of MT070 relative to the other Cyg OB2

\footnotetext{
${ }^{3}$ http://www.chara.gsu.edu/ gudehus/binary.html
} 
members.

Figure 2 displays an average of ten WIRO spectra of MT070 (top) covering the red spectral range and six WIYN spectra (bottom) covering the blue spectral range. Labels denote key spectral features. The composite WIYN spectrum for MT070 shows He II $\lambda 4200: H e$ I $\lambda 4144$, one of the primary temperature-sensitive line ratios in this wavelength regime, in a ratio of $1: 1$, indicating the temperature class of the primary is approximately O9(Sota et al. 2011), in agreement with the initial estimate of Kiminki et al. (2007). Additionally, the luminosity-sensitive ratio Si IV $\lambda$ 4089:He I $\lambda 4026$ appears in a ratio of approximately 3:4, indicating an evolved luminosity class of III-II (Sota et al. 2011). In the composite WIRO spectrum, C III $\lambda 5696$ appears to be slightly in emission, O III $\lambda 5592$ :He II $\lambda 5411$ appears in a ratio of approximately $3: 5$, and C IV $\lambda 5801$ :He II $\lambda 5411$ appears in a ratio of approximately 1:2, also indicating an evolved type (Walborn 2009). We therefore adopt the spectral type of O9III for the primary of MT070. This is consistent with the relative faintness $(V=12.99)$ but the larger extinction of $\Delta A_{V}=+1.8 \mathrm{mag}$ (inferred from $B-V=2.10 \mathrm{mag}$ ) relative the average Cyg OB2 member $(B-V \simeq 1.5 \mathrm{mag})$.

Our spectroscopic dataset on MT070 includes 28 epochs covering the period 19992011 from the Keck (HIRES spectrograph; 2 measurements), WIYN (Hydra spectrograph; 5 measurements), and WIRO (WIRO-Longslit spectrograph; 21 measurements) observatories. Our time-series analysis of the MT070 spectra shows a peak in the power spectrum near 6 years, along with several secondary peaks at similarly long periods between 5-8 years. However, the best-fitting orbital solutions invariably converged to a period of $6.19 \pm 0.13$ years, which yielded $\chi_{\text {red }}^{2}=0.68$, considerably smaller than other candidate periods. Figure 3 shows the data and best fitting orbital solution. Filled circles, triangles, and squares denote the Keck, WIYN, and WIRO data, respectively. This solution specifies a systemtic velocity $\gamma=-13 \pm 1 \mathrm{~km} \mathrm{~s}^{-1}$, eccentricity $e=0.34 \pm 0.11$, and a (projected) primary velocity amplitude $K_{1}=9 \pm 1 \mathrm{~km} \mathrm{~s}^{-1}$. Table 3 lists the full orbital parameters for MT070 and other systems. The four data points near phase $\phi=0.9$ are from WIRO in 2010, and, despite the larger uncertainties, these are included to constrain a portion of the orbit otherwise lacking data. The radial velocities reported in Table 2 for data from WIRO have been corrected by -10 $\mathrm{km} \mathrm{s}^{-1}$ to bring it into better agreement with the WIYN data.

If we adopt a primary mass of $M_{1}=22 \mathrm{M}_{\odot}$ based on the O9III spectral type (Martins et al. 2005), we can begin to place constraints on the secondary mass $M_{2}$ and on the inclination of the system. If the inclination of the system were as low as $i=18^{\circ}$, the implied secondary mass approaches that of the primary. Making the motivated assumption that the secondary is on the main sequence, the luminosity of such a star would be smaller than that of the primary, and indeed, we do not see spectral features from the secondary. Therefore, we can 
limit the inclination to $i>18^{\circ}$, making the mass ratio $q=M_{2} / M_{1}<1$ and semi-major axis $a<10.9$ AU. Stronger constraints are not possible because of the low velocity amplitude of the system relative to the spectral resolution of the data. On the other extreme, if $i=90^{\circ}$, the implied secondary mass is $5.0 \mathrm{M}_{\odot}$ (approximately a B6V), $q=0.22$, and $a=9.3$ AU. Photometric surveys of Cyg OB2 (Pigulski \& Kolaczkowski 1998; Henderson et al. 2011) do not list MT070 as an eclipsing system, but, given the long period, the lack of observed eclipses does not place useful constraints on the inclination of the system.

For completeness, we also report briefly the orbital solution that would have resulted without applying the $-10 \mathrm{~km} \mathrm{~s}^{-1}$ systematic velocity correction to the WIRO data. In this case, we find a a similar period but a much more eccentric system: $P=5.79 \pm 0.11 \mathrm{yr}$, $e=0.71 \pm 0.22$, a systemic velocity $\gamma=-1 \pm 4 \mathrm{~km} \mathrm{~s}^{-1}$, and a velocity amplitude $K_{1}=24.4$ $\mathrm{km} \mathrm{s}^{-1}$. The $\chi_{\text {red }}^{2}$ is also considerably larger at 1.12. Consequently, we adopt the $6.19 \mathrm{yr}$ period and solution described previously. In summary, MT070 is a single-lined long-period binary with $P=6.19 \mathrm{yr}, e \simeq 0.34,0.22<q<1$, and $18^{\circ}<i<90^{\circ}$.

\subsection{MT103}

MT103 lies just 195" from MT070, and it is similarly red, with $B-V=2.00$. It is the only double-lined binary reported in this work, but many SB2 systems within Cyg OB2 were described in Papers II-IV. The components are significantly blended, except for the times very near quadrature. We determine velocities using double Guassian fits to the He I $\lambda 5876$ line for the 12 WIRO data (all from 2011) and $\lambda 4471$ for the three WIYN data (two from 2001 and one from 2006). We chose to constrain the width of the lines (we used a FWHM of $3.0 \AA$, estimated from the most deblended spectra) and the ratio of component line depths to be near 2.5:1, within $20 \%$. The power spectrum of the primary exhibited a singular strong peak near 22 days with no credible aliases. The power spectrum of the secondary was consistent with this value but considerably more noisy owing to larger velocity uncertainties. As with MT070, we applied a $-10 \mathrm{~km} \mathrm{~s}^{-1}$ shift to all of the WIRO data to improve agreement with the WIYN velocity scale.

Figure 4 shows the best-fitting orbital solution performed jointly for the primary and secondary stars. The period of $22.104 \pm 0.002$ days is quite secure, anchored by the large time baseline of the combined WIYN and WIRO data. The eccentricity of $e=0.32 \pm 0.05$ is likely to be less secure, owing to the difficulty in resolving the blended spectral features. The velocity semi-amplitudes of $K_{1}=71 \pm 4 \mathrm{~km} \mathrm{~s}^{-1}$ and $K_{2}=100 \pm 8 \mathrm{~km} \mathrm{~s}^{-1}$ for the primary and secondary, respectively, indicate a mass ratio of $0.71 \pm 0.10$. The velocity data for the secondary has large uncertainties, particularly near $\phi=0.6-1$ where the deblending technique 
is only marginally effective. Table 3 includes the full list of orbital elements.

Figure 5 (left panel) displays a portion of the 12 spectra around He I $\lambda 5876$ from the 2011 WIRO observing campaign, ordered by phase. The right panel shows the portion of the spectra surrounding $\mathrm{H} \alpha$. The separation of the components begins near $\phi=0.16$ as the primary becomes blueshifted, and the line separation is well-developed at the level of $\sim 200 \mathrm{~km} \mathrm{~s}^{-1}$ at $0.38<\phi<0.53$. Near $\phi=0.72$ the primary becomes redshifted and the secondary's line is visible on the blueshifted wing until $\phi \simeq 0.95$ or later.

Kiminki et al. (2007) list MT103 as a B1V. A qualitative analysis of the composite of 3 WIYN spectra covering the blue wavelength range, using Grav \& Corbally (2009), supports this classification. In the composite spectrum, the Si IV $\lambda \lambda 4089,4116$ lines are absent. This suggests that the primary component is a B1 or later. Mg II $\lambda 4481$ is still very weak relative to He I $\lambda 4471$, and Si II $\lambda 4128-30$ is also very weak, suggesting that it is earlier than B3. The absence of O II $\lambda 4070$, O II $\lambda 4348$, O II $\lambda 4416$ and N II $\lambda 3995$, which we acknowledge may be lost in the noise of the spectral continua, indicates that the primary is not evolved, and lies in the range $\mathrm{B} 1-2 \mathrm{~V}$. We adopt $\mathrm{B} 1 \mathrm{~V}$ for the primary. The most deblended spectra indicate that the secondary component's He lines are similar to the primary's, but slightly weaker. Given this and that the calculated mass ratio for this system is 0.71 , we adopt B2 as the likely spectral type for the secondary. Our attempt at a spectral synthesis of a B1V (14.2 $\left.\mathrm{M}_{\odot} ; M_{V}=-3.2\right) 4$ and a B2V $\left(10.9 \mathrm{M}_{\odot} ; M_{V}=-2.45\right)$ provides reasonable agreement with the most deblended spectra and is consistent with the measured mass ratio. This suggests a luminosity ratio of about $2: 1$ and a mass ratio consistent with the measured 0.71 value. These masses imply an inclination of $i \simeq 50^{\circ}$ in order to match the observed radial velocities. The resulting semi-major axis of the systems is then $a \simeq 0.45 \mathrm{AU}$.

The measured systemic velocity of $-28 \pm 3 \mathrm{~km} \mathrm{~s}^{-1}$ is noteworthy for being about 15 $\mathrm{km} \mathrm{s}^{-1}$ more negative than the mean of $V_{\text {helio }}=-13 \mathrm{~km} \mathrm{~s}^{-1}$ for massive stars in Cyg OB2 (Kiminki et al. 2007). Without the $10 \mathrm{~km} \mathrm{~s}^{-1}$ velocity offset applied to the WIRO data, the systemic velocity for MT103 would be in better agreement with the rest of Cyg OB2 massive stars. We note here that the uncertainty on the systemic velocity is likely to be somewhat larger than the formal $3 \mathrm{~km} \mathrm{~s}^{-1}$ error quoted and that we do not consider the difference from $-13 \mathrm{~km} \mathrm{~s}^{-1}$ to be significant, given possible systematic velocity uncertainties at the 10-12 $\mathrm{km} \mathrm{s}^{-1}$ level.

\footnotetext{
${ }^{4}$ Interpolated from Drilling \& Landolt (2000).
} 


\subsection{MT174}

Kiminki et al. (2007) classify MT174 as a B2IV, while Massey \& Thompson (1991) estimated B2V. Figure 6 displays an average spectrum of MT174 using ten 2011 WIRO data (top) covering the red spectral range and using seven WIYN spectra (bottom) covering the blue spectral range. Labels mark key spectral features. The primary temperaturesensitive line ratio in this wavelength regime is Si IV $\lambda 4089$ :Si III $\lambda 4552$ Gray \& Corbally (2009). Unfortunately, the composite WIYN spectrum does not cover Si III $\lambda 4552$. However, the spectrum does show very weak Si IV $\lambda 4089$ and no Si IV $\lambda 4116$, suggesting a temperature class of B1-B2. The He I $\lambda 4471: \mathrm{Mg}$ II $\lambda 4481$ temperature-sensitive ratio, which becomes useful around B2-B3 is $\sim 1: 5$ and indicates a temperature class of B2 or earlier (Walborn \& Fitzpatrick 1990). Additionally, He I $\lambda 4009$ and He I $\lambda 4026$ are quite strong and in a ratio of 1:2 respectively, also indicating a temperature class of approximately B2 (Walborn \& Fitzpatrick 1990). The composite WIYN and WIRO spectra show narrow Balmer line widths. In conjunction with the weak, but clearly present N II $\lambda 3995$ and O II $\lambda \lambda 4070,4076$, this suggests a luminosity class of III. Finally, a direct comparison to HD35468 (B2III) from the Walborn \& Fitzpatrick (1990) digital atlas provides the best agreement with both the composite WIYN and WIRO spectra. Therefore, we adopt a spectral type of B2III for the primary component of MT174. At $\mathrm{V}=12.55$ and $\mathrm{B}-\mathrm{V}=1.21$, it is not unusually colored for early type stars in Cyg OB2, but it is 1-2 magnitudes brighter than other B2 stars in our CygOB2 sample, consistent with an evolved luminosity class.

Our 10 WIRO spectra from 2011 reveal that this is a single-lined spectroscopic binary with a period of $4.536 \pm 0.020 \mathrm{~d}, e=0.53 \pm 0.13$, and $K_{1}=9 \pm 1 \mathrm{~km} \mathrm{~s}^{-1}$. Figure 7 shows the data and best fitting orbital solution, which has $\chi_{r e d}^{2}=0.71$. The systemic velocity of $+11 \pm 2$ $\mathrm{km} \mathrm{s}^{-1}$ is considerably more positive than the $-13 \mathrm{~km} \mathrm{~s}^{-1}$ average of probable Cyg OB2 members (Kiminki et al. 2007). This may be the result of systematic differences between the WIRO data and that from other observatories. Our attempts to include the seven measurements from WIYN over the period 2001 - 2006 resulted in poor or non-convergent solutions, even after applying a $-10--20 \mathrm{~km} \mathrm{~s}^{-1}$ offset to the WIRO measurements. This may indicate the presence of additional kinematic variations in the MT174 system, possibly the result of the gravitational influence of a third body on timescales of many months or years. Unfortunately, our most reliable data do not have sufficient time coverage to constrain the nature of possible additional velocity perturbations in MT174. Given that the mean velocity measured in our WIYN spectra is $V_{\text {helio }}=-6 \mathrm{~km} \mathrm{~s}^{-1}$ compared to +12 for the WIRO data, we apply a $-18 \mathrm{~km} \mathrm{~s}^{-1}$ zero-point shift to our final orbital solution, summarized in Table 3 and the radial velocities in Table 2. The resulting systemic velocity of $-6 \mathrm{~km} \mathrm{~s}^{-1}$ is more consistent with the ensemble of Cyg OB2 stars. 
If we adopt a primary mass appropriate to a B2III $\left(M \simeq 10.9 \mathrm{M}_{\odot} ; R=10 R_{\odot}\right)$, the implied secondary mass is $M_{2}=0.35 \mathrm{M}_{\odot}$ for $i=90^{\circ}$ and $M_{2}=1.0 \mathrm{M}_{\odot}$ for $i=20^{\circ}$. For inclinations as low as $3^{\circ} M_{2}$ approaches $M_{1}$, a lower bound imposed by the lack of secondary spectral features. The absence of known eclipses (Henderson et al. 2011), coupled with the narrow linewidth of $\sim 1 \AA$ FWHM for He I $\lambda 4471$ in our single 1999 Keck spectrum, is consistent with a small projected rotational velocity, which would imply a small inclination angle if the components' spin axes are aligned with the orbital axis. (The small linewidth is also consistent with the evolved luminosity classification.) The inclination is, effectively, unconstrained, and may lie in the range $3^{\circ}<i<87^{\circ}$. For all inclinations greater than about 15 degrees, the secondary is $\mathrm{M}<1.4 \mathrm{M}_{\odot}$, making MT174 one of the most extreme mass ratio $(q<0.13)$ systems yet uncovered in Cyg OB2.

MT174 is an interesting system in that it is the first binary in the Cyg OB2 radial velocity survey to contain a massive star and a quasi solar-mass star. As such, it is a potential progenitor of a low-mass X-ray binary system (LMXRB). The distribution of mass ratios among Cyg OB2 stars is approximately flat, and some $45 \%$ of massive binary systems have components capable of mass transfer, (Kiminki \& Kobulnicky 2012), meaning that 5$10 \%$ of massive binaries may be progenitors of low-mass X-ray binaries. However, the relative paucity of observed LMXRBs in nature implies that the formation channels for such objects are narrow and require special conditions during post-main-sequence evolution, as discussed by van den Heuvel (1983).

\section{4. $\quad \mathrm{MT267}(\mathrm{A} 11)$}

Figure 8 shows the 25 WIRO data, all from 2011 June - 2011 November, and the orbital solution for the SB1 system MT267 (A11 in the notation of Comerón et al. 2002). The period of $P=15.511 \pm 0.056 \mathrm{~d}$, eccentricity of $e=0.21 \pm 0.07$, and velocity amplitude $K_{1}=24 \pm 2 \mathrm{~km} \mathrm{~s}^{-1}$ are well constrained by the data. However, the $\chi_{\text {red }}^{2}$ of 1.59 is larger than for most other systems, suggesting the possibility of photospheric line variations (common for evolved massive stars) or an additional dynamical influence in the system. Our examination of the observed minus computed (O-C) values in Table 2 reveals a weak correlation between Julian date and O-C, but additional data will be needed to establish the nature of additional long-term velocity variations, such as those expected from a third body. The resulting systemic velocity of $\gamma=-13 \pm 1 \mathrm{~km} \mathrm{~s}^{-1}$ is consistent with other CygOB2 stars, and, given the lack of data from other observatories, we do not apply any systematic correction to the velocities plotted in Figure 8 or the data in Table 2. The full suite of orbital parameters appears in Table 3 . 
Figure 9 is an average spectrum of MT267 covering the wavelength range 5400-6700 from our WIRO data. Labels mark key spectral atmospheric and interstellar medium features. The composite spectrum exhibits a He II $\lambda 5411$ :He I $\lambda 5876$ ratio of near unity, indicating a temperature class near O7 (Walborn 1980). O III $\lambda 5592$ is present at roughly 1:3 with He I $\lambda 5876$ and also agrees with a late-O temperature class (Walborn 1980). C III $\lambda 5696$ is prominent in emission. C IV $\lambda \lambda 5801-5812$ and O III $\lambda 5592$ are present at moderate strength and relatively broad, and $\mathrm{H} \alpha$ appears to be partially filled in by emission. This all indicates an evolved star (Walborn 2009, 1980). H $\alpha$ shows variable emission, which is also indicative of an evolved star or an interacting binary. Figure 10 displays a sequence of $\mathrm{H} \alpha$ (right) and He I $\lambda 5876$ (left) spectra, ordered by phase. Changes in the $\mathrm{H} \alpha$ line profile are evident, but He I appears unchanged, except for the periodic velocity variations. Comparing spectral standards from the Jacoby et al. (1984) digital atlas with a composite spectrum of this star, we find that an $\mathrm{O} 7-\mathrm{O} 8$ best fits the temperature of primary component, with the luminosity class in the range III-I. This also agrees with our photometric assessment of this star and is consistent with the spectral type estimated by Negueruela et al. (2008) who give $\mathrm{O} 7.5 \mathrm{Ib}-\mathrm{II}(\mathrm{f})$.

If we adopt a mass of $32 \mathrm{M}_{\odot}$ appropriate for an O7.5III - I (Martins et al. 2005) then the implied secondary mass ranges between $3.0 \mathrm{M}_{\odot}$ for an inclination of $i=90^{\circ}$ and 30 $\mathrm{M}_{\odot}$ for $i=8^{\circ}$ if the secondary approaches the mass of the primary. Given the evolved, luminous nature of the primary, which we assume dominates the luminosity of the system, is it conceivable that the secondary may be as massive as the primary but not visible in the integrated spectrum. The lack of known eclipses (Henderson et al. 2011) provides only a weak constraint that the inclination is smaller than $81^{\circ}$. Thus, we conclude that the mass ratio of the system is $0.09<q<1$ with semi major axis $0.48>a>0.39$ AU. The presence of X-rays and X-ray variability in MT267 (Rauw 2011) is consistent with a wind-wind collision in a close, interacting binary. If the wind collision scenario is correct for the origin of the $\mathrm{X}$-rays, then the secondary component is probably toward the massive end of our allowed range, implying $i<13^{\circ}$ in order to make the mass of the secondary equivalent to a B0V or earlier.

MT267 has an unusually red $B-V$ color (2.19 mag) compared to other massive stars

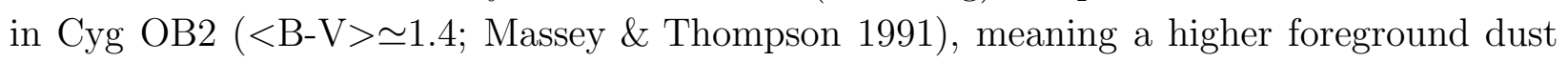
column density toward this star. Figure 11 shows an infrared view of the field around MT267. The left panel is a three-color image with blue/green/red representing the POSS R-band, the Spitzer $4.5 \mu \mathrm{m}$ band, and Spitzer $24 \mu \mathrm{m}$ band. In the right panel blue/green/red represent the UKIDSS JHK images. Labels mark the locations of MT267, and the well-known, heavily reddened mid-B supergiant Cyg OB2 \#12 (MT304). Interestingly, these two evolved massive stars lie within a radius of $53^{\prime \prime}$ from each other. Clearly evident in the left panel is the infrared 
dark cloud adjacent to both objects. This is the Bok globule noted by Scappini et al. (2007) and Poppi et al. (2010). Green contours show the ${ }^{12} \mathrm{CO}$ line intensity integrated over the velocity range $V_{L S R}=9.7-11.7 \mathrm{~km} \mathrm{~s}^{-1}$, corresponding to $V_{\odot}=23.7-25.7 \mathrm{~km} \mathrm{~s}^{-1} .5$ Contour levels show 13, 25, 38, and $50 \mathrm{~K} \mathrm{~km} \mathrm{~s}^{-1}$ intensities corresponding to column densities of $N\left(H_{2}\right)=3.9,7.5,11.4$, and $15.0 \times 10^{21} \mathrm{~cm}^{-2}$, using the mean relation between molecular column density and ${ }^{12} \mathrm{CO}$ integrated intensity $a=3.0 \times 10^{20} \mathrm{~cm}^{2} /\left(\mathrm{K} \mathrm{km} \mathrm{s}^{-1}\right.$ ) (Solomon et al. 1987). Given the conversion factor of Bohlin et a. (1978)6, the contours correspond roughly to extinctions $A_{V}=0.67,1.3,2.0,2.6 \mathrm{mag}$, assuming $R_{V}=3.1$. These are probably lower limits given that ${ }^{12} \mathrm{CO}$ becomes optically thick and/or freezes onto dust grains in the cold cores of molecular clouds. If this Bok globule/molecular cloud lies in the foreground to MT267 (and Cyg OB2\#12), it is conceivably responsible for the higher extinction recorded to these objects. However, the very large extinction of Cyg OB2\#12 ( $\left.A_{V} \sim 12 \mathrm{mag}\right)$ cannot be solely attributed to this cloud. Given the large radial velocity difference between this cloud and Cyg OB2, it is probable that this cloud lies substantially in the foreground. The lack of PAH emission around the borders of this cloud further supports the conjecture that it lies at some distance from the massive ionizing stars.

\subsection{MT734 (Schulte \#11)}

MT734 (VI Cyg \#11/Schulte 11) is a well-known emission-line star of type O5I(f), as classified by Herrero et al. (2002). Figure 1 illustrates that MT734 lies along the western boundary of the Association in an area apparently devoid of $\mathrm{H} \alpha$ and PAH emission, although it is surrounded by molecular clouds. Our 39 spectra, taken with the WIRO Longlit spectrograph over the period 2007-2011, show that this system is a single-lined spectroscopic binary. We find a period of $72.43 \pm 0.07 \mathrm{~d}, e=0.50 \pm 0.06$, and $K_{1}=26 \pm 1 \mathrm{~km} \mathrm{~s}^{-1}$. Figure 12 depicts our best orbital solution for this system. MT734 possesses a systemic velocity of $-31 \pm 1$ $\mathrm{km} \mathrm{s}^{-1}$ which is more negative than most members of the Association. We have not applied any systematic velocity corrections to the WIRO data. The comparatively blue-shifted line centers may be a result of line formation in the upper atmosphere/outflowing wind in this very luminous supergiant.

If we adopt a mass of $51 \mathrm{M}_{\odot}$ appropriate to an O5I $\left(R=19 R_{\odot}\right)$ from Martins et al. (2005), then an inclination of $90^{\circ}$ yields a secondary mass of $M_{2}=7.7 \mathrm{M}_{\odot}, q=0.15$, and

\footnotetext{
${ }^{5}$ The conversion from the local standard of rest (LSR) to heliocentric reference frame is $V_{\odot}=V_{L S R}+14$ $\mathrm{km} \mathrm{s}^{-1}$ in the direction of CygOB2.

${ }^{6} \frac{A_{V}}{R_{V}}=<E(B-V)>(\mathrm{mag})=N\left(H+H_{2}\right) \mathrm{cm}^{2} / 5.8 \times 10^{21}\left(\mathrm{~cm}^{2} \mathrm{mag}^{-1}\right)$
} 
$a=1.31 \mathrm{AU}$. Assuming $i=13^{\circ}$ implies that the seondary mass approaches that of the primary, $q \simeq 1$ and $a=1.51 \mathrm{AU}$. The lack of known eclipses does not place helpful constraints on the inclination. MT734 is listed as a $1400 \mathrm{MHz}$ radio continuum source with $S_{1400}=0.5 \pm 0.3 \mathrm{mJy}$ (Setia Gunawan et al. 2003). This could result from the coliding winds of two massive stars.

Figure [13] shows the spectral time sequence for MT734, ordered by phase, in the spectral region around He I $\lambda 5876$ (left panel) and $\mathrm{H} \alpha$ (right panel). The He I line shows a smooth progression of central wavelength with orbital phase, while the $\mathrm{H} \alpha$ line is broad, exhibits emission, and has irregular line profiles, typical of supergiants.

\section{Discussion and Summary}

We have presented orbital solutions for five additional massive binaries that are probable members of the Cygnus OB2 Association, bringing the total number of multiple systems therein to 25. The vast majority of these have complete orbital solutions, making this the largest collection of massive binary statistics in any single cluster or association to date. Kiminki \& Kobulnicky (2012) have used these data, along with previously known systems documented in Papers I-IV, to infer the underlying distribution of periods, mass ratios,

and eccentricities for Cyg OB2 as a whole. The analysis of Kiminki \& Kobulnicky (2012) suggests that the known list of 25 binaries out of 114 Cyg OB2 systems surveyed is reasonably complete at periods less than about 30 days, but that we should expect to discover an additional 10-15 binaries having longer periods as the Cygnus OB2 Radial Velocity Survey continues.

Noteworthy among the new list of massive binaries is the discovery of a probable progenitor of a low-mass X-ray binary system (MT174). Also noteworthy is the long-period system MT070 with a period of 6.2 years. Both of these low-amplitude systems $\left(K_{1} \simeq 9 \mathrm{~km} \mathrm{~s}^{-1}\right)$ attest to the capabilities of the Survey to achieve orbital solutions for either low-inclination or low- $q$ systems.

We are grateful to Gregor Rauw who made us aware of the possible binary nature of MT267. We thank the time allocation committees of the Lick, Keck, WIYN, and WIRO observatories for granting us observing time and making this project possible. The efforts of WIRO staff James Weger and Jerry Bucher made this science possible. We acknowledge continued support from the National Science Foundation through Research Experience for Undergraduates (REU) program grant AST 03-53760, through grant AST 03-07778, and through grant AST 09-08239, and the support of the Wyoming NASA Space Grant Consortium through grant \#NNX10A095H. 
Facilities: WIRO (), WIYN (), Keck:I ()

\section{REFERENCES}

Bohlin, R. C., Savage, B. D., \& Drake, J. F. 1978, ApJ, 224, 132

Burrows, A., Hayes, J., \& Fryxell, B. A. 1995, ApJ, 450, 830

Blaauw, A. 1961, BAN, 15, 265

Caballero-Nieves, S. M., Gies, D. R., Roberts, L. C., \& Turner, N. H. 2011, Bulletin de la Societe Royale des Sciences de Liege, 80, 639

Comerón, F., Pasquali, A., Rodighiero, G., et al. 2002, A\&A, 389, 874

Contreras, M. E., Rodriguez, L. F., Tapia, M., Cardini, D., Emanuele, A., Badiali, M., \& Persi, P. 1997, ApJ, 488, 153

De Becker, M., Rauw, G., \& Manfroid, J. 2004, A\&A, 424, L39

Drilling, J. S., \& Landolt, A. U. 2000, in Astrophysical Quantities, ed. A. N. Cox (4th ed.; New York; Springer), 381

Eldridge, J. J., Izzard, R. G., \& Tout, C. A. 2008, MNRAS, 384, 1109

Garmany, C. D., Conti, P. S., \& Massey, P. 1980, ApJ, 242, 1063

Setia Gunawan, D. Y. A., de Bruyn, A. G., van der Hucht, K. A., \& Williams, P. M. 2003, ApJS, 149, 123

Gies, D. R., \& Bolton, C. T. 1986, ApJS, 61, 419

Gray, R. O., \& Corbally, C. J. 2009, in Stellar Spectral Classification, ed. Spergel, D. N. (1st ed.; New Jersey, Princeton University Press), 115

Hall, D. S. 1974, AcA, 24, 69

Hanson, M. M. 2003, ApJ, 597, 957

Henderson, C. B., Stanek, K. Z., \& Prieto, J. L. 2011, ApJ, 194, 27

Herrero, A., Puls, J., \& Najarro, F. 2002, A\&A, 396, 949

Izzard, R.G., Ramirez-Ruiz, E, Tout, C.A. 2004, MNRAS, 348, 1215 
Jacoby, G. H. \& Hunter, D. A. 1984, ApJS, 56, 257

Jenniskens, P., \& Désert, F. X. 1994, Astronomy \& Astrophysics Suppliment Series, 106, 39

Kiminki, D. C., et al. 2007, ApJ, 664, 1120 (Paper I)

Kiminki, D. C., McSwain, M. V., \& Kobulnicky, H. A. 2008, ApJ, 679, 1478 (Paper II)

Kiminki, D. C., Kobulnicky, H. A., Gilbert, I., Bird, S., Chunev, G. 2009, AJ, 137, 4608 (Paper III)

Kiminki, D. C., Kobulnicky, H. A., Ewing, I., et al. 2012, ApJ, 747, 41 (Paper IV)

Kiminki, D. C., \& Kobulnicky, H. A. 2012, ApJ, 751, 4 (Paper V)

Kobulnicky, H. A., \& Fryer, C. L. 2007, ApJ, 670, 747

Krumholz, M. R., Cunningham, A. J., Klein, R. I., \& McKee, C. F. 2010, ApJ, 713, 1120

Krumholz, M. R., \& Thompson, T. A. 2007, ApJ, 661, 1034

Hubeny, I., \& Lanz, T. 1995, ApJ, 439, 875

Lanz, T., \& Hubeny, I. 2003, ApJS, 146, 417

Maeder, A., \& Meynet, G. 2000, ARA\&A, 38, 143

Markwardt, C. B. 2009, Astronomical Data Analysis Software and Systems XVIII, 411, 251

Martins, F., Schaerer, D., \& Hillier, D. J. 2005, A\&A, 436, 1049

Massey, P., \& Thompson, A. B. 1991, AJ, 101, 1408

Miczaika, G. R. 1953, PASP, 65, 141

Nazé, Y., De Becker, M., Rauw, G., \& Barbieri, C. 2008, A\&A, 483, 543

Nazé et al. 2010, ApJ, 719, 634

Negueruela, I., Marco, A., Herrero, A., \& Clark, J. S. 2008, A\&A, 487, 575

Otero, S. 2008a, Open European Journal on Variable Stars, 83, 1

Otero, S. 2008b, Open European Journal on Variable Stars, 91, 1

Pigulski, A., \& Kolaczkowski, Z. 1998, MNRAS, 298, 753 
Poppi, S., Scappini, F., Cecchi-Pestellini, C., \& Maccaferri, G. 2010, MNRAS, 407, 1255

Rauw, G., Vreux, J. M., \& Bohannan, B. 1999, ApJ 517, 416

Rauw, G. 2011, A\&A, 536, A31

Rios, L. Y., \& DeGioia-Eastwood, K. 2004, BAAS, 205, No. 09.05

Roberts, D. H., Lehár, J., \& Dreher, J. W. 1987, AJ, 93, 968

Romano, G. 1969, MmSAI, 40, 375

Salpeter, E. E. 1955, ApJ, 121, 161

Sana, H., \& Evans, C. J. 2011, IAU Symposium, 272, 474

Scappini, F., Cecchi-Pestellini, C., Casu, S., \& Olberg, M. 2007, A\&A, 466, 243

Schulte, D. H. 1958, AJ, 128, 41

Smith, R. J., Longmore, S., \& Bonnell, I. 2009, MNRAS, 400, 1775

Solomon, P. M., Rivolo, A. R., Barrett, J., \& Yahil, A. 1987, ApJ, 319, 730

Sota, A., Maíz Apellániz, J., Walborn, N. R., Alfaro, E. J., Barbá, R. H., Morrell, N. I., Gamen, R. C., \& Arias, J. I. 2011, 193, 24

Stroud, V. E., Clark, J.S., Negueruela, I. , Roche, P., \& Norton, A.J. 2009, A\&A, 511, 84

van den Heuvel, E. P. J. 1983, Accretion-Driven Stellar X-ray Sources, 303

Walborn, N. R. 1973, ApJ, 180, L35

Walborn, N. R. 1980, ApJS, 44, 535

Walborn, N. R. 2009, in Stellar Spectral Classification, ed. Spergel, D. N. (1st ed.; New Jersey, Princeton University Press), 66

Walborn, N. R., \& Fitzpatrick, E. L. 1990, PASP, 102, 379

Wilson, O. C. 1948, PASP, 60, 385

Wilson, O. C., \& Abt, A. 1951, ApJ, 144, 477

Woosley, S. E., \& Bloom, J. S. 2006, ARA\&A, 44, 507

Woosley, S. E., Langer, N., \& Weaver, T. A. 1993, ApJ, 411, 823 
Wozniak, P. R., et al. 2004, AJ, 127, 2436, Northern Sky Variability Survey: Public Data Release 
Table 1. Ephemeris for MT103

\begin{tabular}{|c|c|c|c|c|c|c|}
\hline Date (HJD-2,400,000) & $\phi$ & $\begin{array}{c}V_{r 1} \\
\left(\mathrm{~km} \mathrm{~s}^{-1}\right)\end{array}$ & $\begin{array}{c}O_{1}-C_{1} \\
\left(\mathrm{~km} \mathrm{~s}^{-1}\right)\end{array}$ & $\begin{array}{c}V_{r 2} \\
\left(\mathrm{~km} \mathrm{~s}^{-1}\right)\end{array}$ & $\begin{array}{c}\mathrm{O}_{2}-C_{2} \\
\left(\mathrm{~km} \mathrm{~s}^{-1}\right)\end{array}$ & Observatory \\
\hline \multicolumn{7}{|c|}{ MT103 } \\
\hline $52146.75 \ldots$ & 0.660 & $-12.0(5.0)$ & 1.5 & $-179.0(50.0)$ & -128.6 & WIYN \\
\hline $52162.75 \ldots \ldots \ldots \ldots \ldots \ldots$ & 0.383 & $-102.9(8.0)$ & -6.5 & $28.0(19.0)$ & -37.4 & WIYN \\
\hline $53989.75 \ldots \ldots \ldots \ldots \ldots \ldots$ & 0.036 & $18.0(9.0)$ & 7.4 & $-186.0(33.0)$ & -102.0 & WIYN \\
\hline $54696.75 \ldots \ldots$ & 0.031 & $7.0(6.0)$ & -4.3 & $-117.0(23.0)$ & -32.0 & WIRO \\
\hline $54697.75 \ldots$ & 0.075 & $6.0(4.0)$ & 1.2 & $-142.0(45.0)$ & -66.1 & WIRO \\
\hline $55779.75 \ldots \ldots \ldots \ldots \ldots \ldots$ & 0.018 & $0.0(9.0)$ & -12.8 & $-99.0(50.0)$ & -11.9 & WIRO \\
\hline $55791.50 \ldots \ldots \ldots \ldots \ldots \ldots$ & 0.551 & $-55.0(7.4)$ & 27.5 & $41.0(13.0)$ & -5.0 & WIRO \\
\hline $55805.50 \ldots \ldots \ldots \ldots \ldots$ & 0.186 & $-16.8(12.9)$ & 2.2 & $-55.0(14.0)$ & -12.2 & WIRO \\
\hline $55832.50 \ldots \ldots \ldots \ldots \ldots$ & 0.403 & $-107.0(4.4)$ & -1.7 & $71.1(9.0)$ & -6.8 & WIRO \\
\hline $55834.50 \ldots \ldots \ldots \ldots \ldots \ldots$ & 0.495 & $-117.7(6.7)$ & -2.0 & $80.5(12.0)$ & -11.8 & WIRO \\
\hline $55855.25 \ldots \ldots \ldots \ldots \ldots \ldots$ & 0.441 & $-113.4(6.9)$ & 5.0 & $90.0(11.0)$ & -6.1 & WIRO \\
\hline $55857.50 \ldots \ldots \ldots \ldots \ldots \ldots$ & 0.535 & $-99.0(5.1)$ & -4.4 & $84.7(11.0)$ & 21.8 & WIRO \\
\hline $55866.50 \ldots \ldots \ldots \ldots \ldots \ldots$ & 0.941 & $32.5(7.6)$ & 12.6 & $-76.0(34.0)$ & 21.0 & WIRO \\
\hline $55901.00 \ldots \ldots \ldots \ldots \ldots \ldots$ & 0.511 & $-107.0(5.0)$ & 2.1 & $101.0(13.0)$ & 17.9 & WIRO \\
\hline $55906.25 \ldots \ldots \ldots \ldots \ldots \ldots$ & 0.744 & $16.0(11.5)$ & 4.3 & $-92.8(38.5)$ & -7.3 & WIRO \\
\hline
\end{tabular}


Table 2. Ephemerides for MT070, MT174, MT267, \& MT734

\begin{tabular}{|c|c|c|c|c|}
\hline Date (HJD-2,400,000) & $\phi$ & $\begin{array}{c}V_{r 1} \\
\left(\mathrm{~km} \mathrm{~s}^{-1}\right)\end{array}$ & $\begin{array}{c}O_{1}-C_{1} \\
\left(\mathrm{~km} \mathrm{~s}^{-1}\right)\end{array}$ & Observatory \\
\hline \multicolumn{5}{|c|}{ MT070 } \\
\hline $51467.00 \ldots$ & 0.165 & $-19.7(2.1)$ & -1.5 & KECK \\
\hline $51806.00 .$. & 0.315 & $-8.7(1.8)$ & 2.7 & KECK \\
\hline $52146.75 \ldots$ & 0.465 & $-7.3(5.4)$ & 0.7 & WIYN \\
\hline $52161.75 \ldots$ & 0.472 & $-8.8(4.4)$ & -0.9 & WIYN \\
\hline $52162.75 \ldots \ldots \ldots \ldots \ldots \ldots$ & 0.472 & $-10.0(4.5)$ & -2.1 & WIYN \\
\hline $53340.50 \ldots$ & 0.994 & $-26.4(6.3)$ & -2.4 & WIYN \\
\hline $53989.75 \ldots$ & 0.281 & $-18.0(9.9)$ & -5.5 & WIYN \\
\hline $54399.75 \ldots \ldots \ldots \ldots \ldots \ldots$ & 0.462 & $-9.1(4.8)$ & -1.1 & WIRO \\
\hline $54401.75 \ldots \ldots \ldots \ldots \ldots \ldots$ & 0.463 & $-16.1(3.3)$ & -8.1 & WIRO \\
\hline $54402.75 \ldots \ldots \ldots \ldots \ldots \ldots$ & 0.464 & $-6.8(3.6)$ & 1.2 & WIRO \\
\hline $54410.75 \ldots \ldots$ & 0.467 & $-8.1(4.2)$ & -0.2 & WIRO \\
\hline $54696.75 \ldots$ & 0.594 & $-5.2(2.9)$ & 1.7 & WIRO \\
\hline $54697.75 \ldots$. & 0.594 & $-7.7(3.3)$ & -0.8 & WIRO \\
\hline $54698.75 \ldots$ & 0.595 & $-6.5(2.7)$ & 0.4 & WIRO \\
\hline $54757.75 \ldots$ & 0.621 & $-6.3(2.8)$ & 0.6 & WIRO \\
\hline $55404.75 \ldots$ & 0.907 & $-9.1(8.6)$ & 7.4 & WIRO \\
\hline $55422.00 \ldots \ldots \ldots \ldots \ldots \ldots$ & 0.915 & $-10.8(9.3)$ & 6.3 & WIRO \\
\hline $55468.75 \ldots \ldots \ldots \ldots \ldots \ldots$ & 0.935 & $-26.1(12.2)$ & -7.0 & WIRO \\
\hline $55491.50 \ldots \ldots \ldots \ldots \ldots \ldots$ & 0.945 & $-22.0(3.4)$ & -1.9 & WIRO \\
\hline $55716.75 \ldots \ldots \ldots \ldots \ldots \ldots$ & 0.045 & $-24.4(2.6)$ & 0.5 & WIRO \\
\hline $55718.75 \ldots \ldots \ldots \ldots \ldots \ldots$ & 0.046 & $-24.1(2.4)$ & 0.8 & WIRO \\
\hline $55766.75 \ldots \ldots \ldots \ldots \ldots \ldots$ & 0.067 & $-23.9(2.2)$ & 0.2 & WIRO \\
\hline $55805.50 \ldots \ldots$ & 0.084 & $-23.2(2.2)$ & 0.0 & WIRO \\
\hline $55832.50 \ldots \ldots$ & 0.096 & $-26.1(2.5)$ & -3.6 & WIRO \\
\hline $55834.50 \ldots \ldots \ldots \ldots \ldots \ldots$ & 0.097 & $-22.2(2.1)$ & 0.3 & WIRO \\
\hline $55847.50 \ldots$. & 0.103 & $-22.0(2.1)$ & 0.1 & WIRO \\
\hline $55855.25 \ldots \ldots \ldots \ldots \ldots \ldots$ & 0.106 & $-22.7(2.6)$ & -0.9 & WIRO \\
\hline $55866.50 \ldots \ldots \ldots \ldots \ldots \ldots$ & 0.111 & $-19.7(2.2)$ & 1.9 & WIRO \\
\hline \multicolumn{5}{|c|}{ MT174 } \\
\hline $55727.00 \ldots$ & 0.974 & $7.3(4.3)$ & -1.2 & WIRO \\
\hline $55759.00 \ldots \ldots \ldots \ldots \ldots \ldots$ & 0.029 & $16.7(1.9)$ & -2.1 & WIRO \\
\hline $55781.00 \ldots \ldots \ldots \ldots \ldots \ldots$ & 0.879 & $6.0(2.9)$ & 3.0 & WIRO \\
\hline $55791.50 \ldots \ldots \ldots \ldots \ldots \ldots$ & 0.194 & $19.7(3.1)$ & 2.0 & WIRO \\
\hline $55832.50 \ldots \ldots \ldots \ldots \ldots \ldots$ & 0.233 & $15.0(2.6)$ & -1.5 & WIRO \\
\hline $55836.50 \ldots \ldots \ldots \ldots \ldots \ldots$ & 0.115 & $20.9(2.5)$ & 0.7 & WIRO \\
\hline $55855.50 \ldots \ldots \ldots \ldots \ldots \ldots$ & 0.304 & $13.4(2.8)$ & -1.2 & WIRO \\
\hline $55857.50 \ldots \ldots \ldots \ldots \ldots \ldots$ & 0.745 & $4.8(2.6)$ & -0.6 & WIRO \\
\hline $55876.25 \ldots \ldots \ldots \ldots \ldots \ldots$ & 0.879 & $1.0(2.6)$ & -2.1 & WIRO \\
\hline $55906.25 \ldots \ldots \ldots \ldots \ldots \ldots$ & 0.493 & $11.2(2.5)$ & 0.8 & WIRO \\
\hline
\end{tabular}


Table 2-Continued

\begin{tabular}{|c|c|c|c|c|}
\hline Date (HJD-2,400,000) & $\phi$ & $\begin{array}{c}V_{r 1} \\
\left(\mathrm{~km} \mathrm{~s}^{-1}\right)\end{array}$ & $\begin{array}{c}O_{1}-C_{1} \\
\left(\mathrm{~km} \mathrm{~s}^{-1}\right)\end{array}$ & Observatory \\
\hline \multicolumn{5}{|c|}{ MT267 } \\
\hline $55713.75 \ldots$ & 0.950 & $19.2(4.2)$ & 4.9 & WIRO \\
\hline $55714.75 \ldots \ldots \ldots \ldots \ldots \ldots$ & 0.009 & $7.7(4.0)$ & -0.9 & WIRO \\
\hline $55715.75 \ldots \ldots \ldots$ & 0.075 & $-7.1(4.2)$ & -3.2 & WIRO \\
\hline $55716.75 \ldots \ldots \ldots \ldots \ldots \ldots$ & 0.138 & $-16.2(4.3)$ & -0.1 & WIRO \\
\hline $55717.75 \ldots \ldots \ldots \ldots \ldots \ldots$ & 0.202 & $-19.1(4.3)$ & 6.2 & WIRO \\
\hline $55718.75 \ldots \ldots \ldots \ldots \ldots \ldots$ & 0.269 & $-25.2(4.3)$ & 5.5 & WIRO \\
\hline $55726.75 \ldots$ & 0.783 & $6.1(4.0)$ & 5.7 & WIRO \\
\hline $55727.75 .$. & 0.850 & $6.1(3.2)$ & -2.8 & WIRO \\
\hline $55737.75 \ldots \ldots \ldots \ldots \ldots \ldots$ & 0.493 & $-27.5(4.2)$ & 1.3 & WIRO \\
\hline $55738.75 \ldots \ldots \ldots \ldots \ldots \ldots$ & 0.558 & $-19.3(3.3)$ & 5.1 & WIRO \\
\hline $55739.50 \ldots \ldots$ & 0.601 & $-24.3(16.7)$ & -3.5 & WIRO \\
\hline $55740.50 \ldots$ & 0.665 & $-12.7(3.7)$ & 1.6 & WIRO \\
\hline $55741.50 \ldots \ldots$ & 0.735 & $-11.1(3.3)$ & -5.1 & WIRO \\
\hline $55758.75 \ldots \ldots \ldots \ldots \ldots \ldots$ & 0.846 & $13.3(3.0)$ & 5.0 & WIRO \\
\hline $55765.75 \ldots \ldots \ldots \ldots \ldots \ldots$ & 0.294 & $-35.8(4.2)$ & -4.0 & WIRO \\
\hline $55767.50 \ldots \ldots$ & 0.406 & $-41.0(5.0)$ & -8.7 & WIRO \\
\hline $55768.50 \ldots \ldots$ & 0.472 & $-30.6(4.8)$ & -0.8 & WIRO \\
\hline $55779.75 \ldots \ldots$ & 0.192 & $-34.7(5.6)$ & -10.6 & WIRO \\
\hline $55779.75 \ldots \ldots$ & 0.200 & $-18.3(4.8)$ & 6.8 & WIRO \\
\hline $55780.75 \ldots$ & 0.257 & $-35.8(5.9)$ & -5.8 & WIRO \\
\hline $55781.75 \ldots$ & 0.321 & $-37.9(9.1)$ & -5.4 & WIRO \\
\hline $55790.50 \ldots \ldots \ldots$ & 0.889 & $6.5(3.8)$ & -6.1 & WIRO \\
\hline $55834.50 \ldots \ldots \ldots \ldots \ldots \ldots$ & 0.725 & $-10.1(4.0)$ & -2.8 & WIRO \\
\hline $55857.25 \ldots \ldots \ldots$ & 0.199 & $-26.5(4.3)$ & -1.6 & WIRO \\
\hline $55866.25 \ldots \ldots \ldots \ldots \ldots \ldots$ & 0.781 & $1.5(4.5)$ & 1.5 & WIRO \\
\hline \multicolumn{5}{|c|}{ MT734 } \\
\hline $54347.75 \ldots$ & 0.025 & $-22.8(10.0)$ & -1.1 & WIRO \\
\hline $54348.75 \ldots \ldots \ldots \ldots \ldots \ldots$ & 0.039 & $-17.4(10.0)$ & -1.4 & WIRO \\
\hline $54399.75 \ldots \ldots \ldots \ldots \ldots \ldots$ & 0.743 & $-61.2(16.7)$ & -16.3 & WIRO \\
\hline $54402.75 \ldots \ldots \ldots \ldots \ldots$ & 0.785 & $-42.2(8.3)$ & 6.1 & WIRO \\
\hline $54403.50 \ldots \ldots \ldots \ldots \ldots \ldots$ & 0.795 & $-54.9(10.0)$ & -5.8 & WIRO \\
\hline $54403.75 \ldots \ldots \ldots \ldots \ldots \ldots$ & 0.798 & $-46.6(10.0)$ & 2.8 & WIRO \\
\hline $54405.75 \ldots \ldots \ldots \ldots \ldots \ldots$ & 0.826 & $-56.3(11.1)$ & -4.5 & WIRO \\
\hline $54642.75 \ldots$ & 0.098 & $-8.8(5.3)$ & -2.6 & WIRO \\
\hline $54644.00 \ldots$ & 0.116 & $-6.1(5.6)$ & -0.1 & WIRO \\
\hline $54644.75 \ldots \ldots \ldots$ & 0.126 & $-6.5(5.3)$ & -0.4 & WIRO \\
\hline $54645.75 \ldots \ldots \ldots \ldots \ldots \ldots$ & 0.140 & $-5.4(5.6)$ & 1.1 & WIRO \\
\hline $54646.75 \ldots \ldots \ldots \ldots \ldots \ldots$ & 0.154 & $-14.3(5.9)$ & -7.3 & WIRO \\
\hline $54647.75 \ldots \ldots \ldots \ldots \ldots \ldots$ & 0.167 & $-0.5(5.3)$ & 7.3 & WIRO \\
\hline $54648.75 \ldots \ldots \ldots \ldots \ldots \ldots$ & 0.181 & $-14.7(5.3)$ & -6.2 & WIRO \\
\hline $54670.00 \ldots \ldots \ldots \ldots \ldots$ & 0.475 & $-30.0(8.3)$ & -3.3 & WIRO \\
\hline
\end{tabular}


Table 2-Continued

\begin{tabular}{|c|c|c|c|c|}
\hline Date (HJD-2,400,000) & $\phi$ & $\begin{array}{c}V_{r 1} \\
\left(\mathrm{~km} \mathrm{~s}^{-1}\right)\end{array}$ & $\begin{array}{c}O_{1}-C_{1} \\
\left(\mathrm{~km} \mathrm{~s}^{-1}\right)\end{array}$ & Observatory \\
\hline $54671.75 \ldots \ldots$ & 0.499 & $-33.6(7.7)$ & -5.3 & WIRO \\
\hline $54696.75 \ldots \ldots \ldots \ldots \ldots \ldots$ & 0.844 & $-62.7(9.1)$ & -9.4 & WIRO \\
\hline $54747.75 \ldots \ldots$ & 0.548 & $-33.5(8.3)$ & -2.2 & WIRO \\
\hline $54748.75 \ldots$ & 0.562 & $-26.4(8.3)$ & 5.8 & WIRO \\
\hline $55404.75 \ldots$ & 0.619 & $-34.6(12.5)$ & 1.3 & WIRO \\
\hline $55409.75 \ldots$ & 0.688 & $-62.8(14.3)$ & -22.1 & WIRO \\
\hline $55421.75 \ldots$ & 0.854 & $-46.7(14.3)$ & 7.4 & WIRO \\
\hline $55422.75 \ldots$ & 0.868 & $-50.1(14.3)$ & 5.1 & WIRO \\
\hline $55423.75 \ldots \ldots \ldots \ldots \ldots \ldots$ & 0.882 & $-46.1(14.3)$ & 10.0 & WIRO \\
\hline $55461.75 \ldots \ldots \ldots \ldots \ldots \ldots$ & 0.406 & $-22.4(20.0)$ & 0.2 & WIRO \\
\hline $55466.75 \ldots \ldots \ldots \ldots \ldots \ldots$ & 0.475 & $-24.8(20.0)$ & 2.0 & WIRO \\
\hline $55498.75 \ldots \ldots$ & 0.917 & $-44.3(20.0)$ & 13.1 & WIRO \\
\hline $55715.00 \ldots$ & 0.903 & $-57.9(5.0)$ & -0.7 & WIRO \\
\hline $55719.00 \ldots \ldots \ldots$ & 0.958 & $-62.8(7.1)$ & -9.9 & WIRO \\
\hline $55737.75 \ldots \ldots \ldots$ & 0.217 & $-10.2(6.2)$ & 0.5 & WIRO \\
\hline $55740.75 \ldots$ & 0.259 & $-7.5(5.9)$ & 5.8 & WIRO \\
\hline $55780.00 \ldots$ & 0.800 & $-54.4(6.7)$ & -4.8 & WIRO \\
\hline $55790.75 \ldots \ldots \ldots \ldots \ldots \ldots$ & 0.949 & $-55.2(5.3)$ & -0.3 & WIRO \\
\hline $55794.50 \ldots \ldots \ldots \ldots \ldots \ldots$ & 0.001 & $-20.7(12.5)$ & 14.1 & WIRO \\
\hline $55805.75 \ldots \ldots$ & 0.156 & $-9.2(5.9)$ & -2.0 & WIRO \\
\hline $55834.50 \ldots \ldots \ldots \ldots \ldots \ldots$ & 0.553 & $-32.5(8.3)$ & -0.9 & WIRO \\
\hline $55847.50 \ldots \ldots \ldots \ldots \ldots \ldots$ & 0.732 & $-44.0(8.3)$ & 0.1 & WIRO \\
\hline $55856.25 \ldots \ldots \ldots \ldots \ldots \ldots$ & 0.853 & $-51.9(8.3)$ & 2.2 & WIRO \\
\hline $55857.50 \ldots \ldots \ldots \ldots \ldots \ldots$ & 0.871 & $-49.9(7.1)$ & 5.5 & WIRO \\
\hline
\end{tabular}


Table 3. Orbital Elements

\begin{tabular}{lrrrrr}
\hline \hline \multicolumn{1}{c}{ Element } & \multicolumn{1}{c}{ MT070 } & \multicolumn{1}{c}{ MT103 } & \multicolumn{1}{c}{ MT174 } & \multicolumn{1}{c}{ MT267 } & \multicolumn{1}{c}{ MT734 } \\
\hline$P($ Days $)$ & $2259 \pm 46$ & $22.104 \pm 0.002$ & $4.536 \pm 0.020$ & $15.511 \pm 0.056$ & $72.43 \pm 0.07$ \\
$e$ & $0.34 \pm 0.11$ & $0.32 \pm 0.05$ & $0.53 \pm 0.13$ & $0.21 \pm 0.07$ & $0.50 \pm 0.06$ \\
$\omega(\mathrm{deg})$ & $157.9 \pm 26$ & $26 \pm 12$ & $282.0 \pm 20$ & $37 \pm 19$ & $262.6 \pm 8$ \\
$\gamma\left(\mathrm{km} \mathrm{s}^{-1}\right)$ & $-13 \pm 1$ & $-28 \pm 3$ & $-6 \pm 2$ & $-13 \pm 1$ & $-31 \pm 1$ \\
$T_{0}\left(\mathrm{HJD}^{-2,400,000)}\right.$ & $53355 \pm 134$ & $55757.2 \pm 0.5$ & $55749.8 \pm 0.1$ & $55745.7 \pm 0.8$ & $55722 \pm 1$ \\
$K_{1}\left(\mathrm{~km} \mathrm{~s}^{-1}\right)$ & $9 \pm 1$ & $71 \pm 4$ & $9 \pm 1$ & $24 \pm 2$ & $26 \pm 1$ \\
$K_{2}\left(\mathrm{~km} \mathrm{~s}^{-1}\right)$ & $\ldots$ & $100 \pm 8$ & $\ldots$ & $\ldots$ & $\ldots$ \\
S. C.1 & O9III & $\mathrm{B} 1 \mathrm{~V}$ & $\mathrm{~B} 2 \mathrm{III}$ & $\mathrm{O} 7.5 \mathrm{III}-\mathrm{I}$ & O5I(f) \\
S. C.2 & $\mathrm{B}:$ & $\mathrm{B} 2 \mathrm{~V}$ & $\ldots$ & $\mathrm{B}-\mathrm{O}$ & early B $-\mathrm{O}$ \\
$q$ & $0.22-1$ & $0.71 \pm 0.10$ & $0.03-0.9$ & $0.09-1$ & $0.15-1$ \\
$i($ degrees $)$ & $90-18$ & $\simeq 50$ & $87-3$ & $81-8$ & $90-13$ \\
$a($ AU) & $9.3-10.9$ & $0.45 \pm 0.02$ & $0.12-0.15$ & $0.39-0.48$ & $1.3-1.5$ \\
$\chi_{\text {red }}^{2}$ & 0.68 & 2.41 & 0.71 & 1.59 & 0.54 \\
\hline
\end{tabular}


Table 4. OB Binaries in Cyg OB2

\begin{tabular}{|c|c|c|c|c|c|c|}
\hline Star & Type & S.C. & $\begin{array}{c}\mathrm{P} \\
\text { (days) }\end{array}$ & $\mathrm{e}$ & $\mathrm{q}$ & Ref. \\
\hline MT059 & SB1 & O8V \& B & $4.8527 \pm 0.0002$ & $0.11 \pm 0.04$ & $0.22-0.67$ & 1 \\
\hline MT070 & SB1 & O9III \& B & $6.19 \mathrm{yr}$ & $0.34 \pm 0.11$ & $0.25-1$ & 0 \\
\hline MT103 & SB2 & $\mathrm{B} 1 \mathrm{~V}+\mathrm{B} 2 ?$ & $22.104 \pm 0.002$ & $0.32 \pm 0.05$ & $0.71 \pm 0.10$ & 0 \\
\hline MT145 & SB1 & O9III \& mid B & $25.140 \pm 0.008$ & $0.291 \pm 0.009$ & $0.26-0.63$ & 2 \\
\hline MT174 & SB1 & B2III \& ?? & $4.536 \pm 0.020$ & $0.53 \pm 0.13$ & $0.03-0.9$ & 0 \\
\hline MT252 & SB2 & B2III \& B1V & $18-19$ & $\ldots$ & $0.8 \pm 0.2$ & 1 \\
\hline MT258 & SB1 & $\mathrm{O} 8 \mathrm{~V} \& \mathrm{~B}$ & $14.660 \pm 0.002$ & $0.03 \pm 0.05$ & $0.18-0.89$ & 1 \\
\hline MT267 & SB1 & O7.5III-I \& O/B & $15.511 \pm 0.056$ & $0.21 \pm 0.07$ & $0.09-1$ & 0 \\
\hline MT311 & SB2 & $\mathrm{B} 2 \mathrm{~V} \& \mathrm{~B} 3 \mathrm{~V}$ & $5.7521 \pm 0.0002$ & $0.02 \pm 0.01$ & $0.8 \pm 0.1$ & 3 \\
\hline MT372 & SB1/EA: & B0V \& B2:V & 2.228 (fixed) & 0.0 (fixed) & $\sim 0.6$ & 2,4 \\
\hline MT421 & SB1:/EA & O9V \& B9V-A0V & 4.161 & $\cdots$ & $\sim 0.16-0.19$ & 5 \\
\hline MT429 & $\mathrm{SB} 1 / \mathrm{EA}$ & B0V \& G0III: & 2.9788 (fixed) & $0.38 \pm 0.08$ & 0.057: & 3,5 \\
\hline MT605 & SB2 & B1V \& B1: & $\sim 4-5$ & $\ldots$ & $0.9 \pm 0.1$ & 3 \\
\hline MT696 & $\mathrm{SB} 2 / \mathrm{EW} / \mathrm{KE}$ & O9.5V \& B0V & $1.4694 \pm 0.0002$ & $0.081 \pm 0.005$ & $0.94 \pm 0.03$ & 3,6 \\
\hline MT720 & SB2 & B0-B1 \& B1-B2 & $4.0677 \pm 0.0003$ & $0.35 \pm 0.02$ & $0.80 \pm 0.08$ & 1,3 \\
\hline MT734 & SB1 & O5I \& O/early B & $72.43 \pm 0.07$ & $0.50 \pm 0.06$ & $0.15-1$ & 0 \\
\hline MT771 & SB2 & O7V \& O9V & $2.82105 \pm 0.00003$ & $0.547 \pm 0.004$ & $0.95 \pm 0.09$ & 1,3 \\
\hline Schulte 3 & SB2/EA: & O6IV: \& O9III & $4.7464 \pm 0.0002$ & $0.070 \pm 0.009$ & $0.44 \pm 0.08$ & 1,7 \\
\hline Schulte 5 & SB2/EB & $\begin{array}{c}\text { O7Ianfp \& Ofpe/WN9 } \\
\text { (\& B0V:) }\end{array}$ & 6.6 (fixed) & 0.0 (fixed) & $0.28 \pm 0.02$ & $\begin{array}{l}8,9,10,11 \\
12,13,14\end{array}$ \\
\hline Schulte $8 \mathrm{a}$ & SB2 & O5.5I \& O6: & 21.908 (fixed) & $0.24 \pm 0.04$ & $0.86 \pm 0.04$ & 15,16 \\
\hline Schulte 9 & SB2 & O5: \& O6-7: & $2.355 \mathrm{yr}$ & $0.708 \pm 0.027$ & $0.9 \pm 0.1$ & 17,18 \\
\hline Schulte 73 & SB2 & O8III \& O8III & $17.28 \pm 0.03$ & $0.169 \pm 0.009$ & $0.99 \pm 0.02$ & 2 \\
\hline A36 & $\mathrm{SB} 2 / \mathrm{EA}$ & B0Ib \& BOIII & $4.674 \pm 0.004$ & $0.10 \pm 0.01$ & $0.70 \pm 0.06$ & $2,19,20$ \\
\hline A45 & SB2 & B0.5V \& B2V:-B3V: & $2.884 \pm 0.001$ & $0.273 \pm 0.002$ & $0.46 \pm 0.02$ & 2,20 \\
\hline B17 & SB2/EB: & O7: \& O9: & $4.0217 \pm 0.0004$ & 0 (fixed) & 0.75 (fixed) & 21,22 \\
\hline
\end{tabular}

Note. - Photometric types EW/KE, EA, and EB stand for eclipsing system of the W UMa type (ellipsoidal; $P<1$ day), Algol type (near spherical), and $\beta$ Lyr type (ellipsoidal; $P>1$ day) respectively. The mass ratio for MT421 is calculated using the O star masses of Martins et al. (2005) and interpolated AB masses of Drilling \& Landolt (2000).

References. - (0) This work; (1) Paper II; (2) Paper III; (3) Paper IV; (4) Wozniak et al. (2004); (5) Pigulski \& Kolaczkowski (1998); (6) Rios \& DeGioia-Eastwood 2004); (7) Kiminki \& Kobulnickv (2012); (8) Wilson (1948); (9)Wilson \& Abt (1951); (10) Miczaika (1953); (11) Walborn (1973); (12) Contreras et al. (1997); (13) Rauw et al. (1999); (14) Hall (1974); (15) Romano (1969); (16) De Becker et al. (2004); (17) Nazé et al. (2008); (18) Nazé et al. (2010); (19) Otero (2008a); (20) Hanson (2003); (21) Stroud et al. (2010); (22) Otero (2008b) 


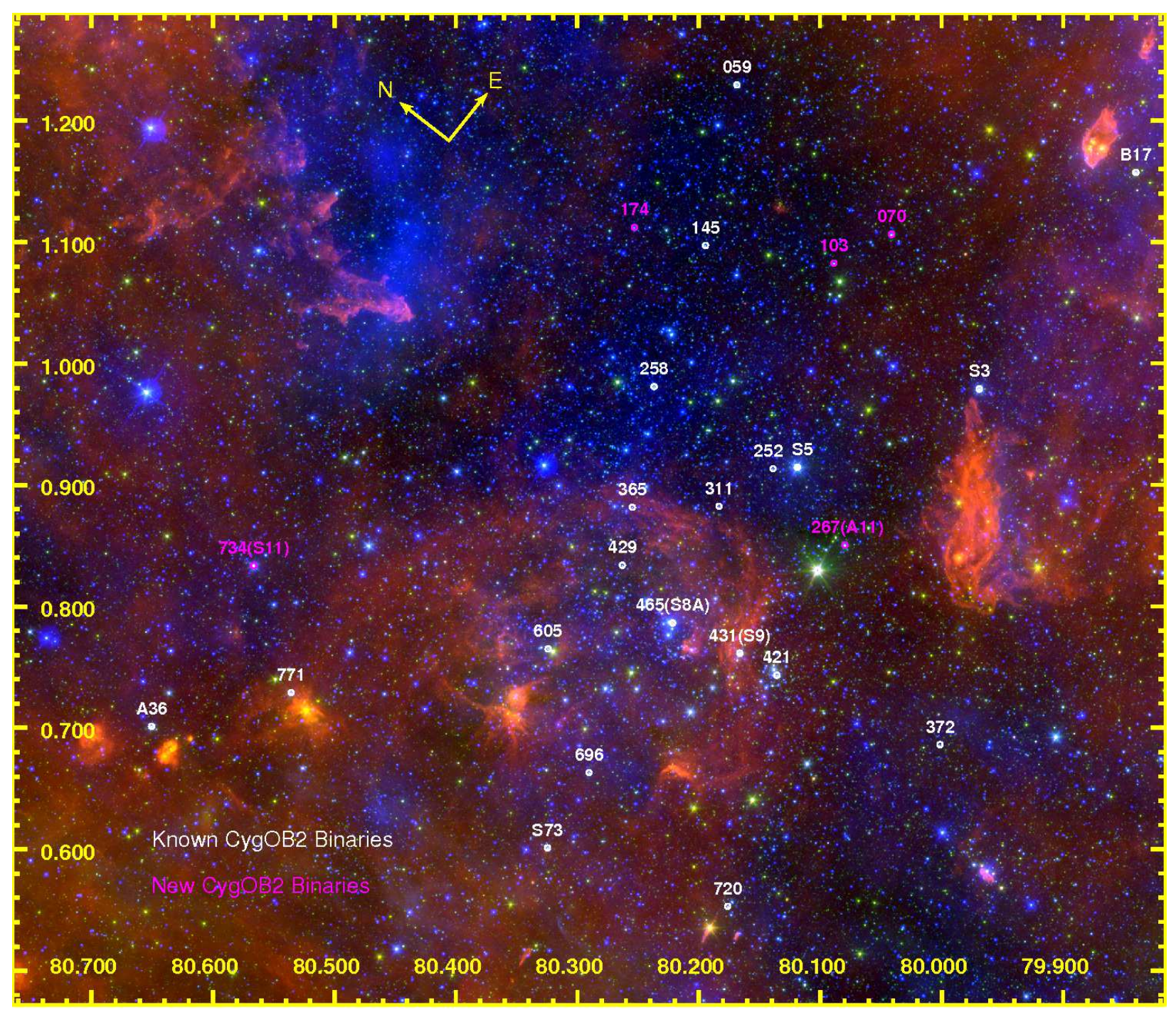

Fig. 1.- Three-color image of the Cygnus OB2 region with the Palomar Sky Survey R image in blue, the Spitzer $4.5 \mu \mathrm{m}$ in green, and Spitzer $8.0 \mu \mathrm{m}$ in red. White labels denote previously known binary systems while magenta labels highlight the newly discovered systems reported herein. Generally, numeration follows the system of Massey \& Thompson (1991), with "S" additionally indicating the numeration of Schulte (1958) and "A" or "B" for that of Comerón et al. (2002). The early-B SB2 system A45 lies just off the field of view to the upper right of B17. 

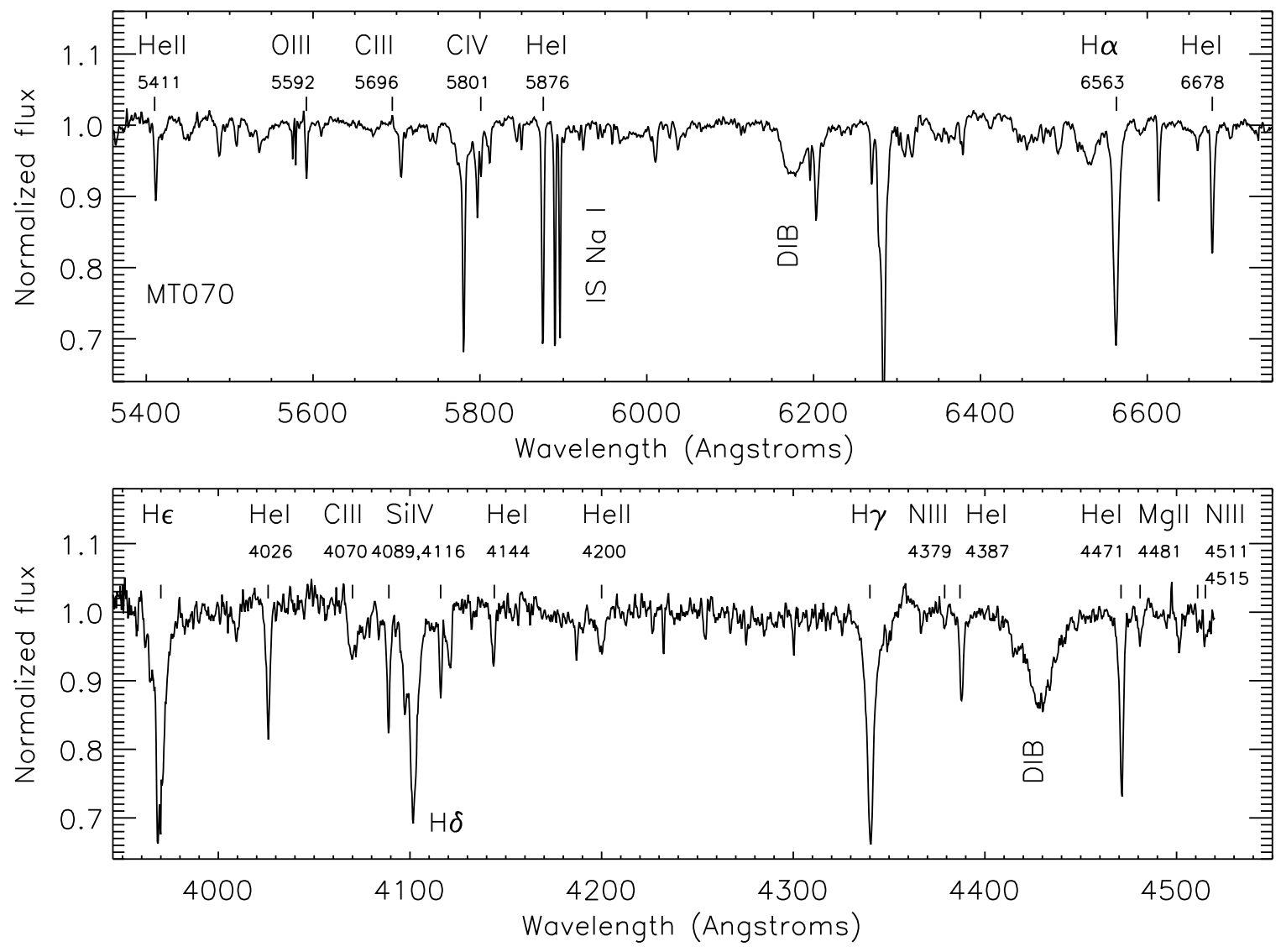

Fig. 2.- Average spectrum of MT070 using data from WIRO (top) and WIYN (bottom). Tickmarks and labels mark key spectral features. Most of the unlabeled absorption features in the upper panel are from diffuse interstellar bands (Jenniskens \& Désert 1994). 


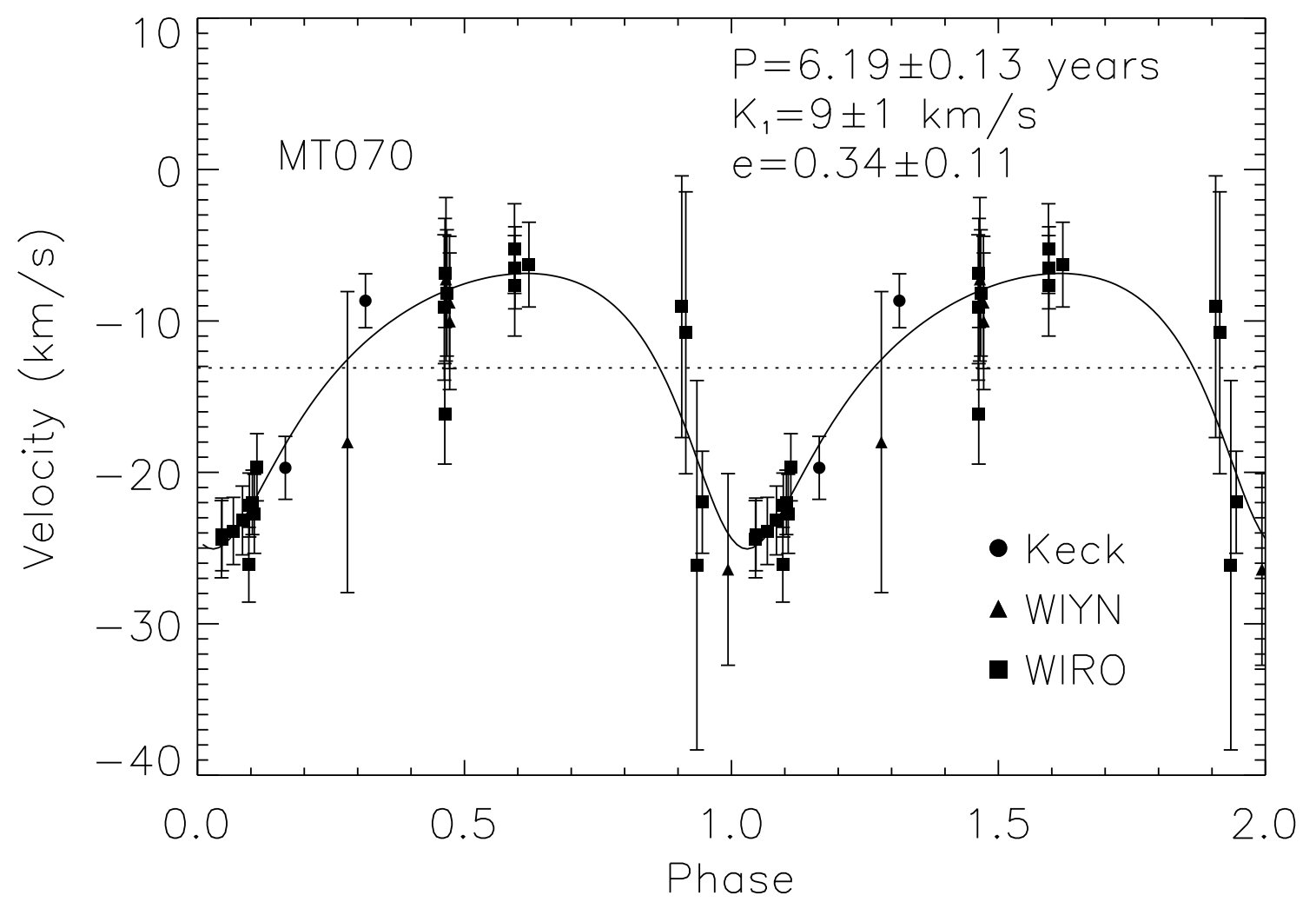

Fig. 3.- Radial velocity data and best-fit orbital solution curve for the 6.19 year SB1 system MT070 (O9III:) using 21 measurements from WIRO (filled squares), five from WIYN (triangles), and two from Keck (circles). The four points with the largest error bars are from the 2010 WIRO campaign which suffered from low spectral resolution. 


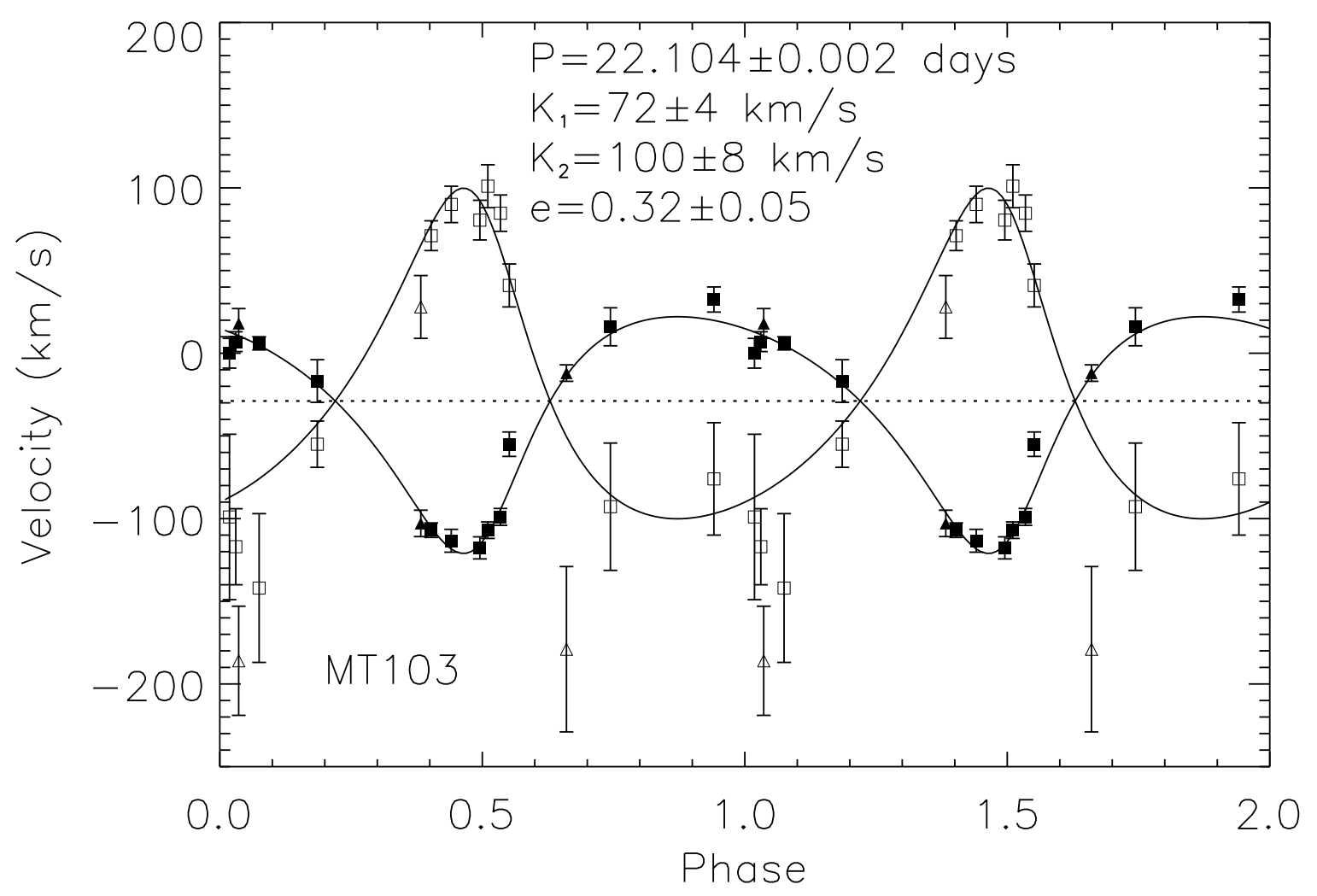

Fig. 4.- Radial velocity data and best-fit orbital solution curve for the 22.10 day SB2 system MT103. Filled symbols denote the primary and open symbols show the secondary. Triangles show WIYN data and squares show WIRO data. 


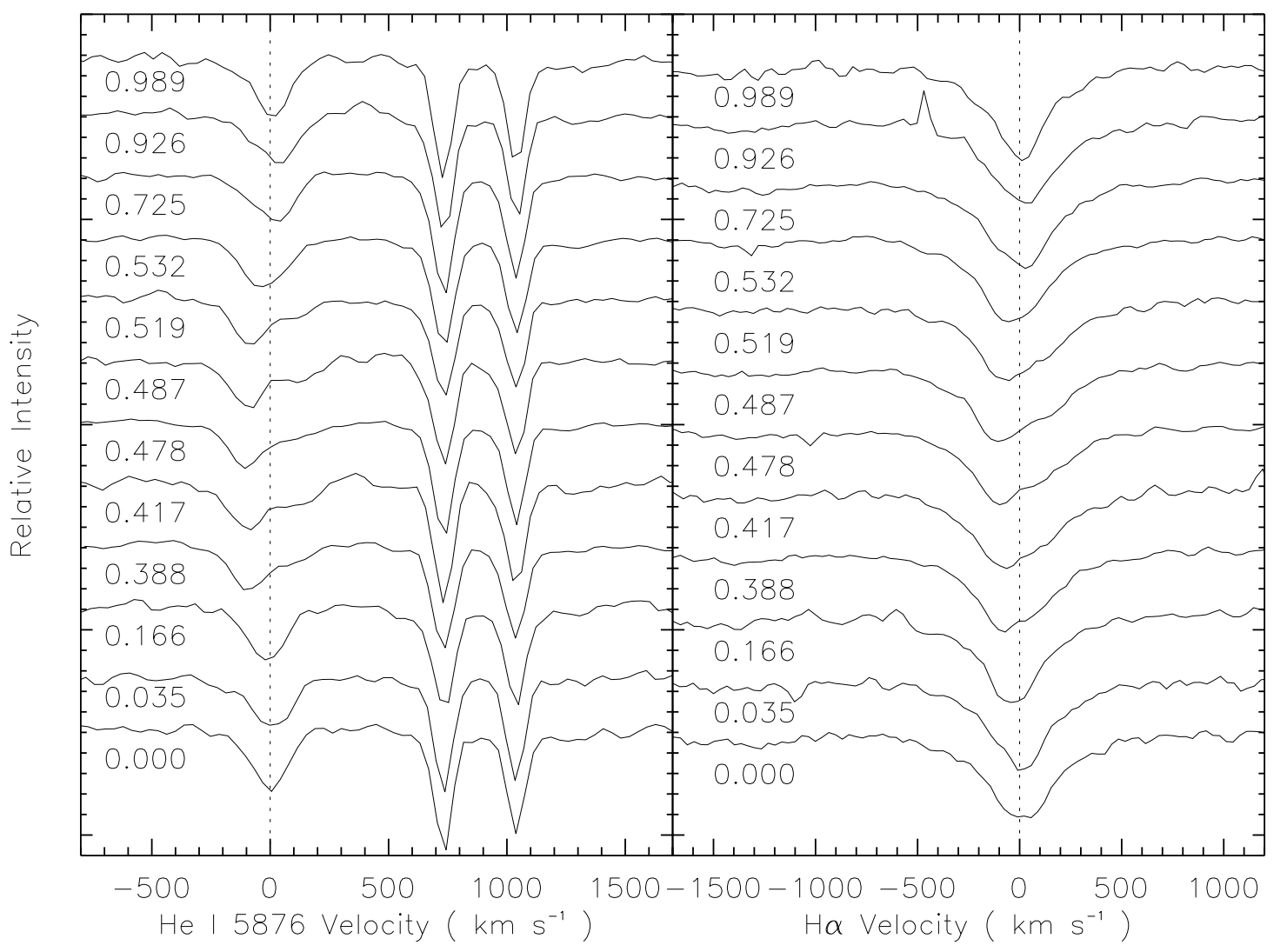

Fig. 5.- A sequence of the 12 WIRO spectra for MT103, ordered by phase, in the spectral region around $\mathrm{He} \mathrm{I} \lambda 5876$ (left) and $\mathrm{H} \alpha$ (right). The $\mathrm{Na} \mathrm{D}$ doublet lies to the right of the He $\lambda 5876$ line. 

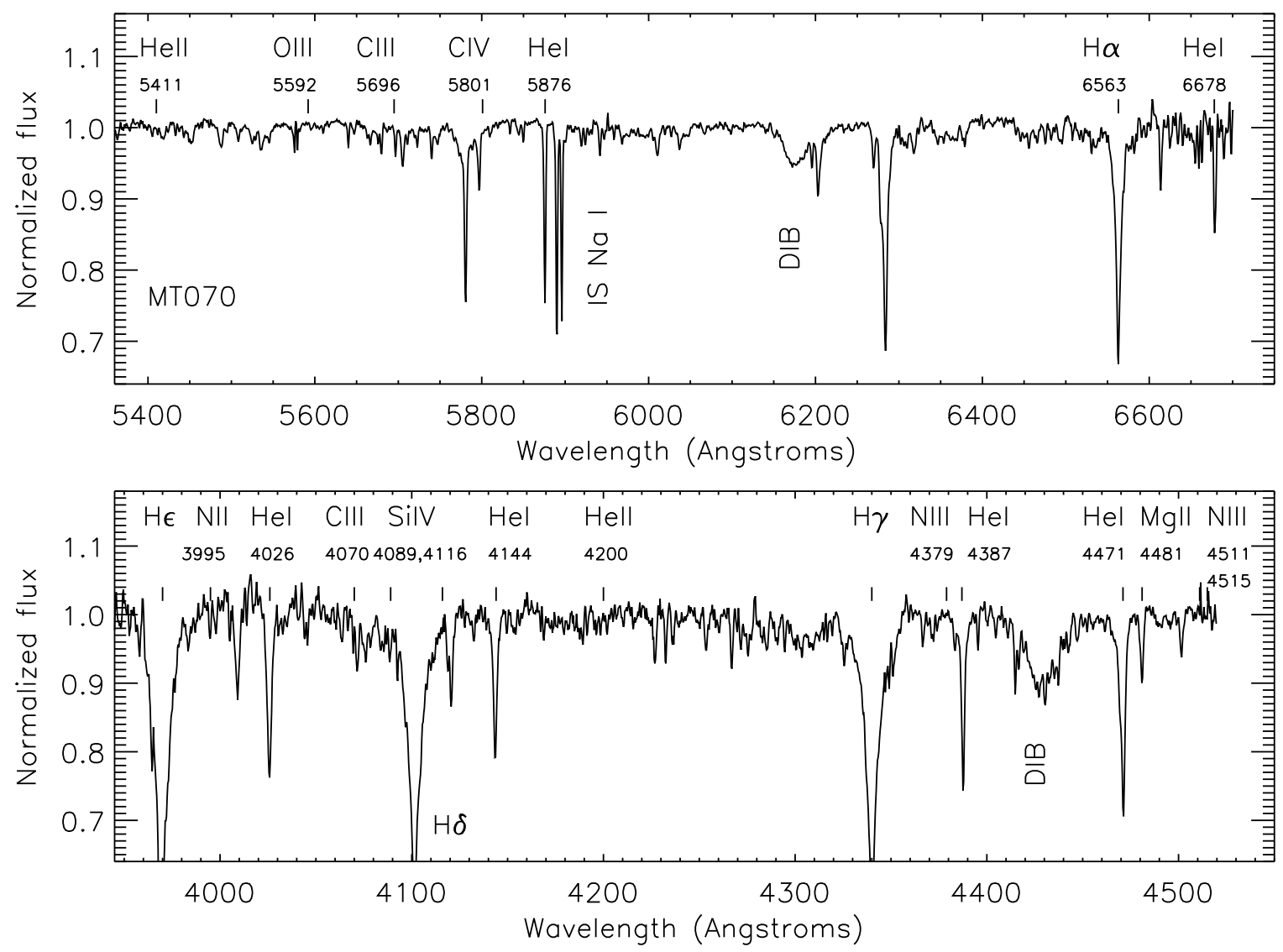

Fig. 6.- Average spectrum of MT174 (B2III) using data from WIRO (top) and WIYN (bottom). 


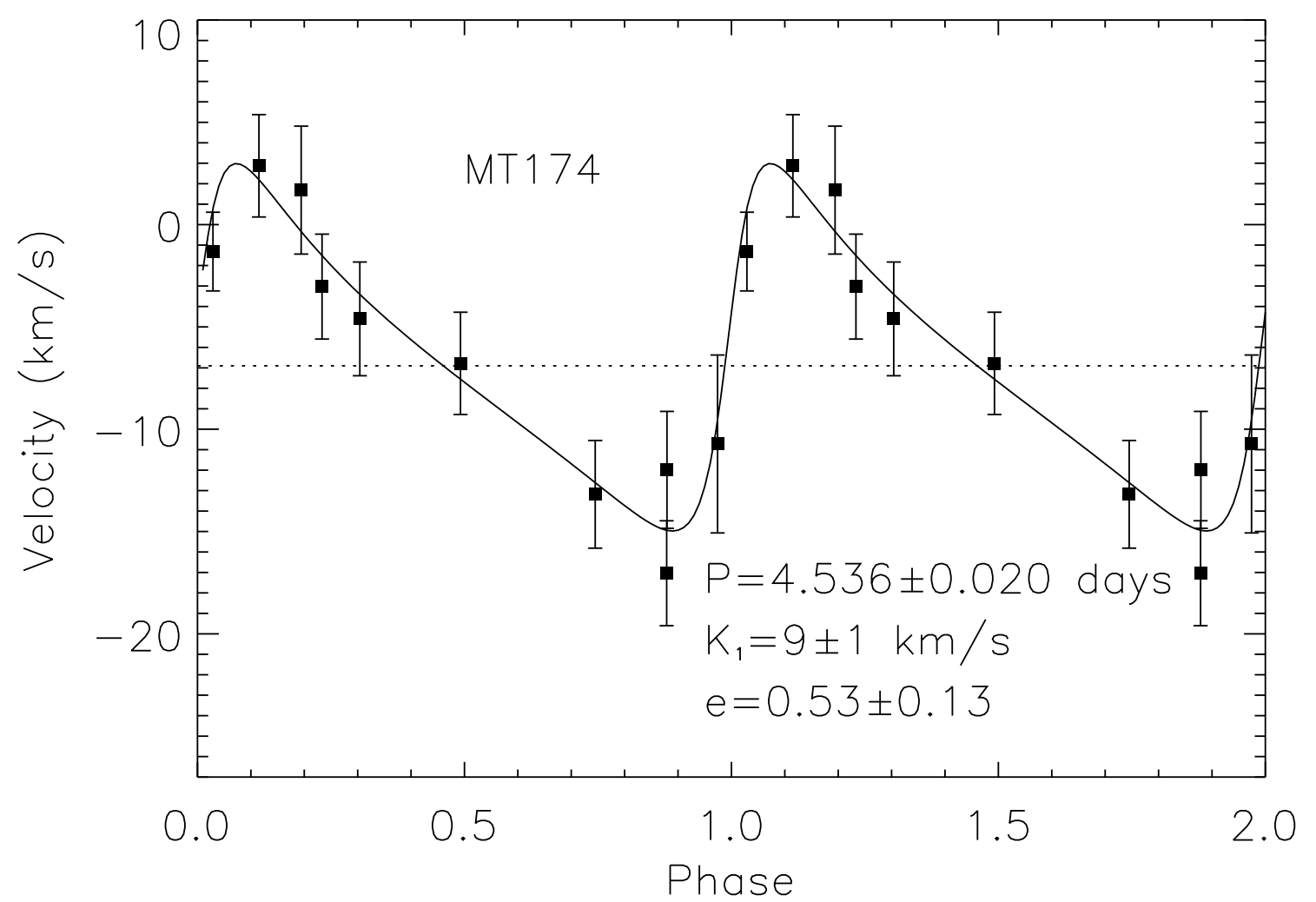

Fig. 7.- Orbital solution for the SB1 system MT174 (B2III) using 10 WIRO data from 2011. 


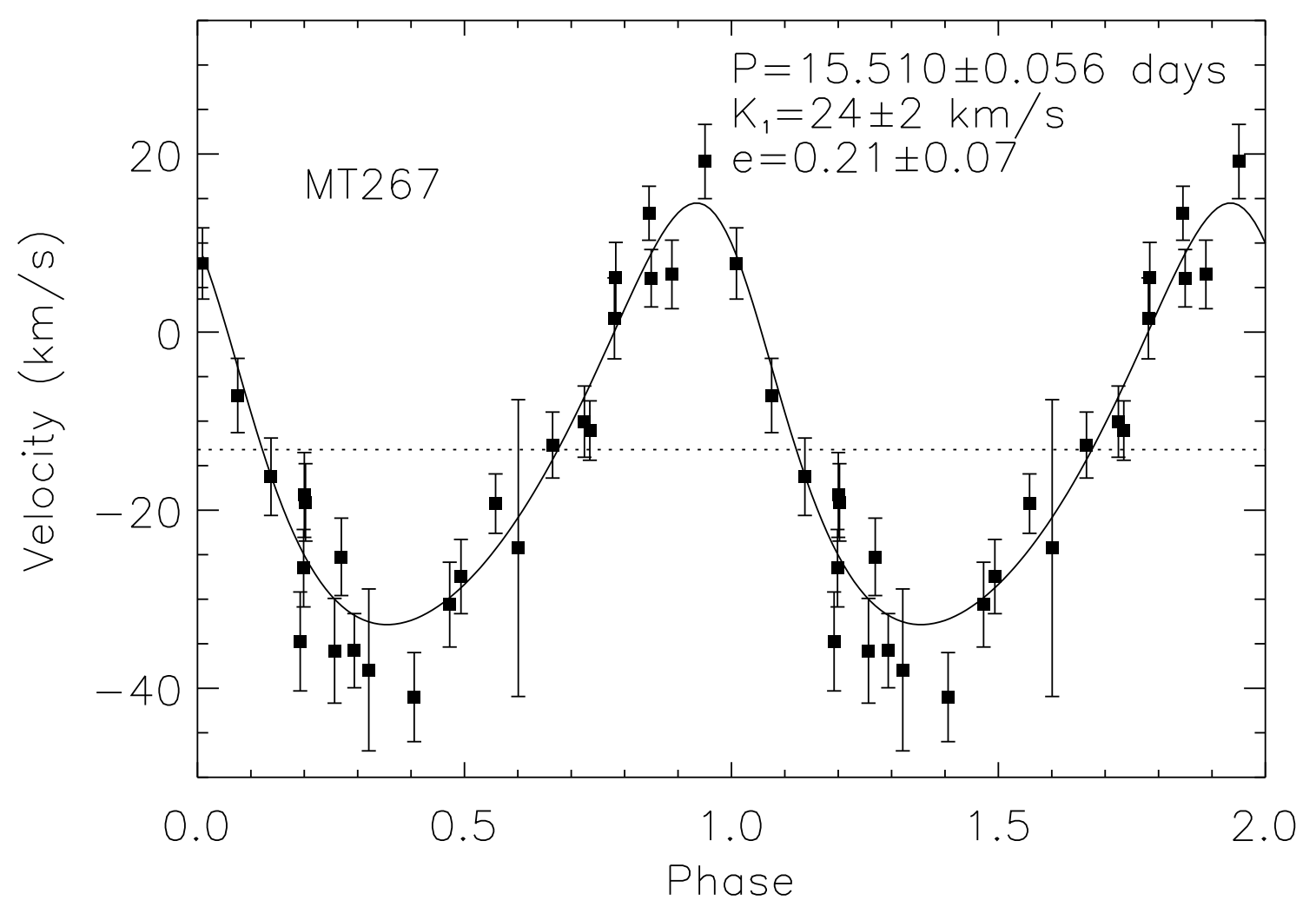

Fig. 8.- Orbital solution for the SB1 system MT267 (O7.5III-I). 


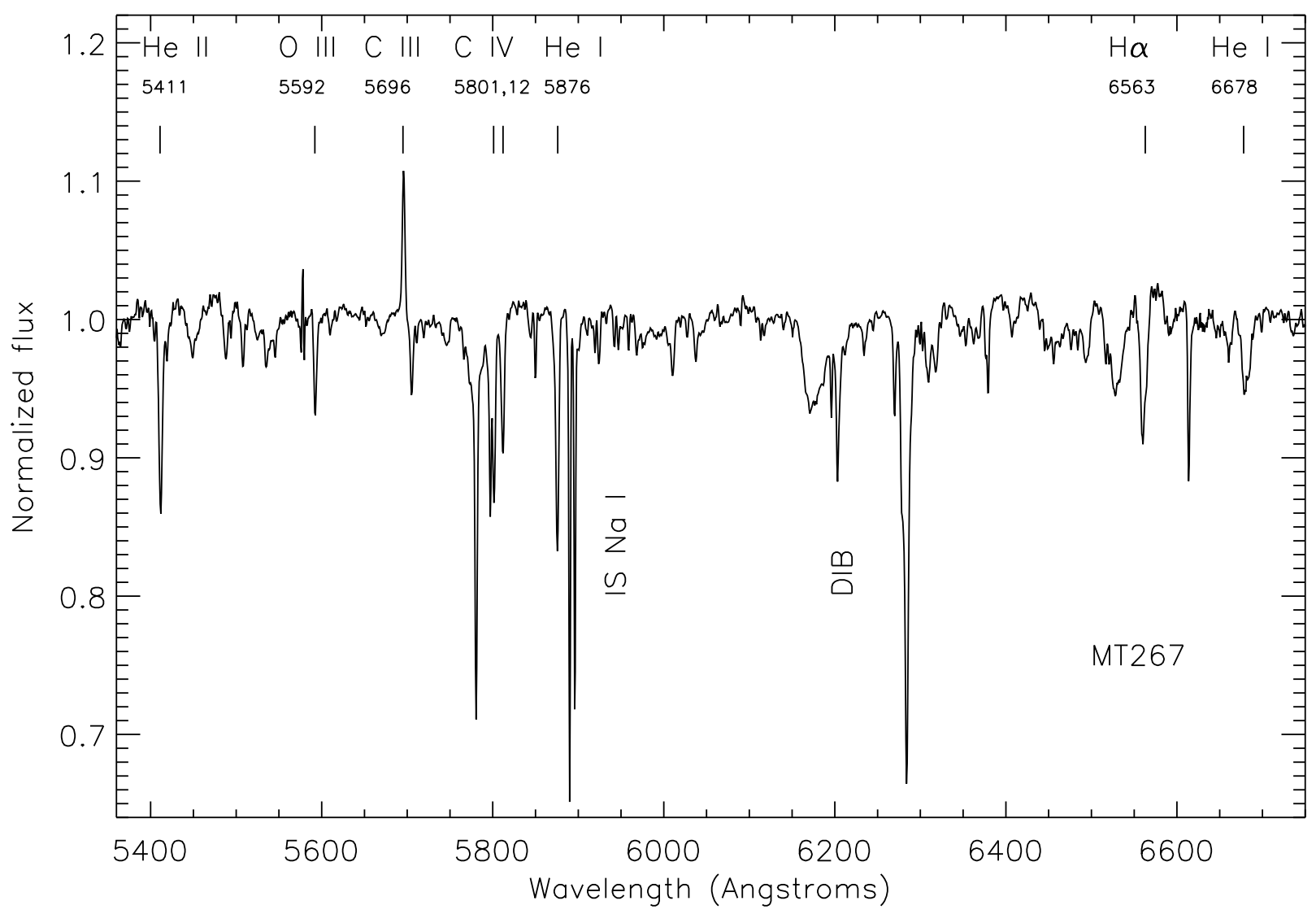

Fig. 9.- Average of 25 WIRO spectra for MT267. Labels mark key spectroscopic features of hot stars along with some prominent insterstellar features. Most of the unlabeled absorption features are from diffuse interstellar bands (Jenniskens \& Désert 1994). 


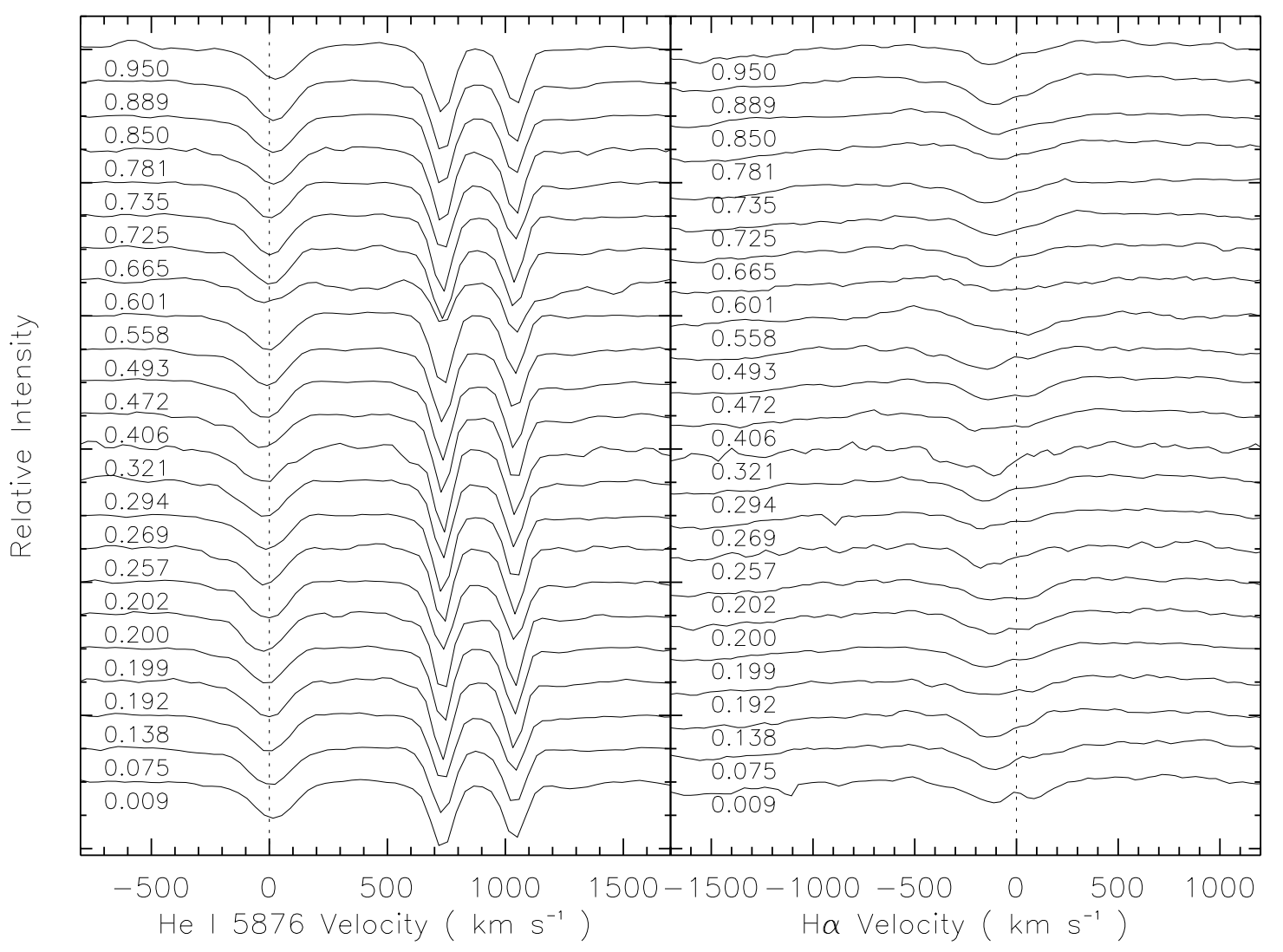

Fig. 10.- A sequence of spectra covering $\mathrm{H} \alpha$ and He I $\lambda 5876$ for MT267, ordered by phase. This O7III: star exhibits variability in the $\mathrm{H} \alpha$ line profile, but not in the He line profiles. 


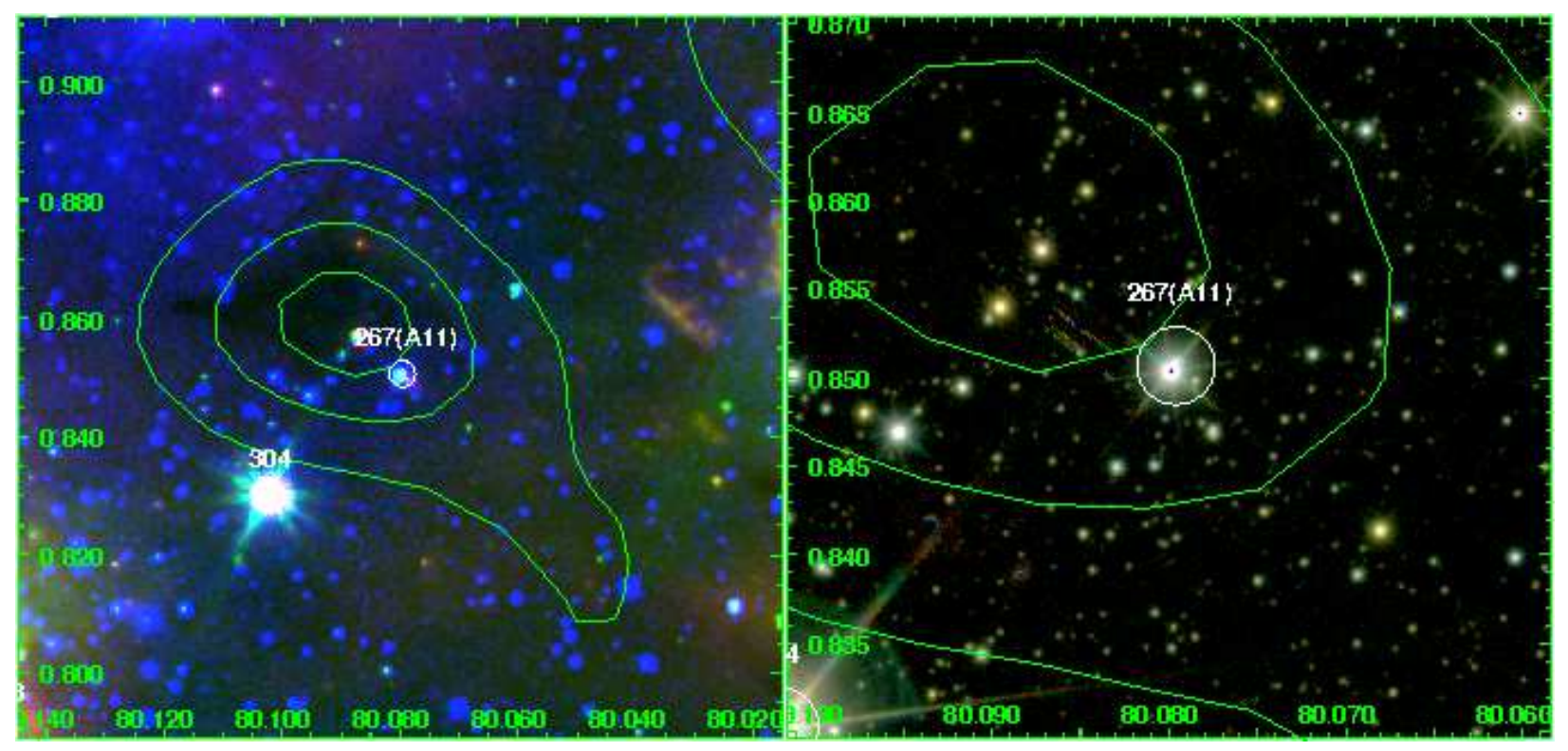

Fig. 11. - The field around MT267. The left panel shows the optical/mid-IR view with POSS R-band in blue and Spitzer GLIMPSE $8 \mu \mathrm{m}$ and $24 \mu \mathrm{m}$ in green and red, respectively. Green contours depict the ${ }^{12} \mathrm{CO}$ globule, which lies coincident with an IR dark cloud. The right panel shows the UKIDSS JHK appearance (blue/green/red) of the field at a higher zoom factor. 


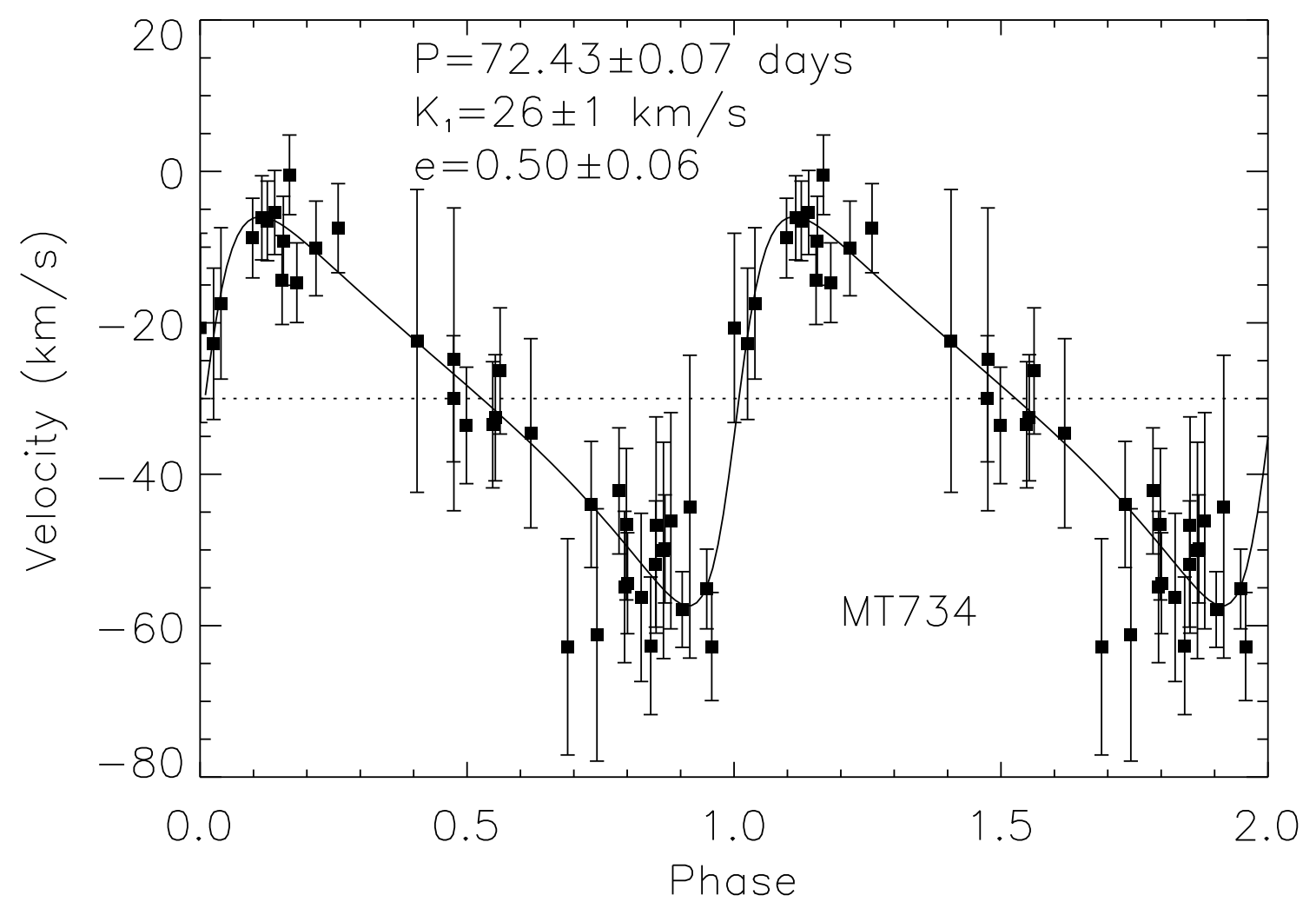

Fig. 12.- Orbital solution for the O5I(f) SB1 system MT734 based on 39 WIRO spectra from 2007-2011. 


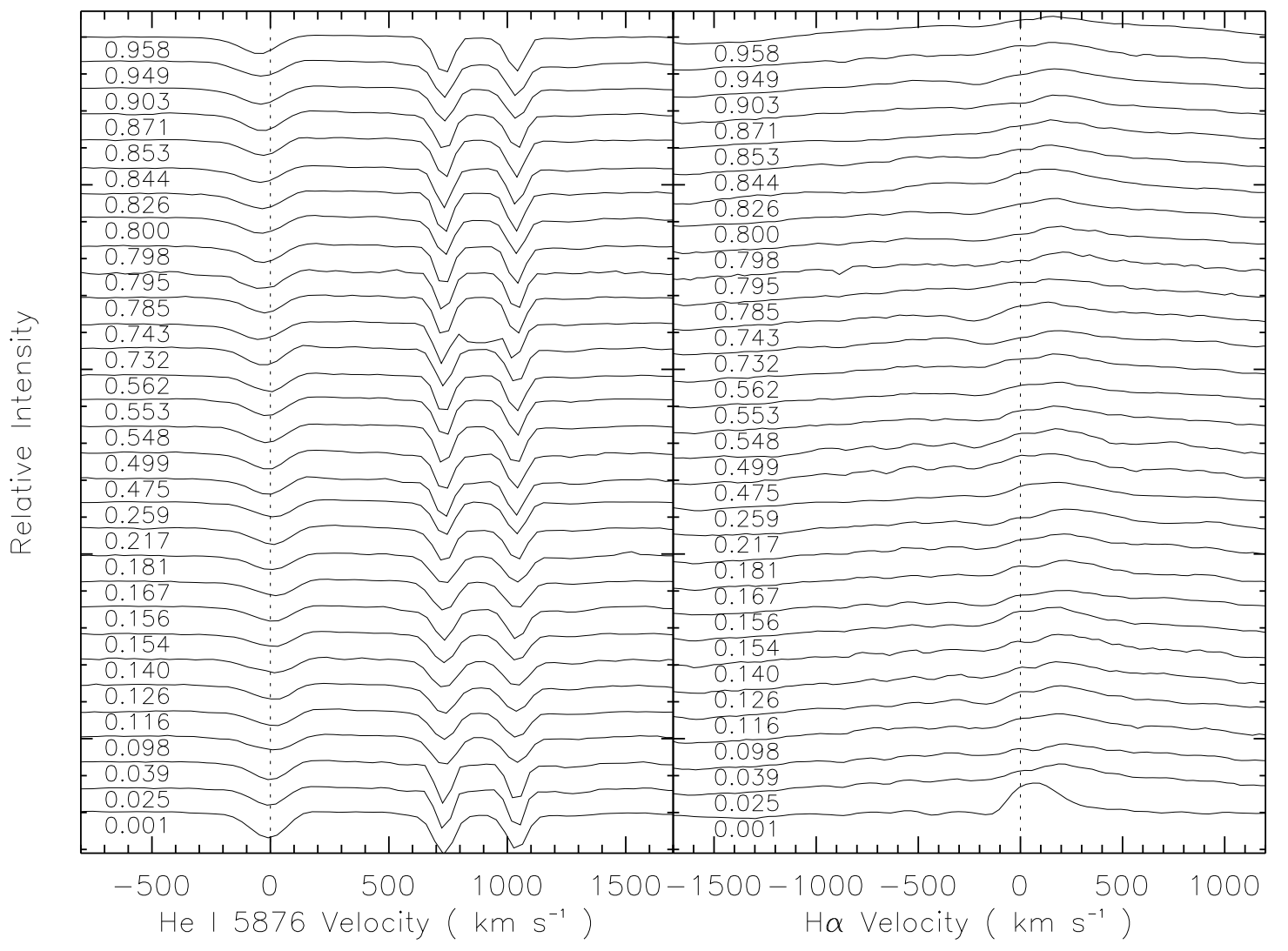

Fig. 13.- A time sequence of spectra for MT734, ordered by phase, in the spectral region around He I $\lambda 5876$ (left panel) and $\mathrm{H} \alpha$ (right panel). 